\title{
PRECIOUS METALS IN SDSS QUASAR SPECTRA. I. TRACKING THE EVOLUTION OF STRONG, $1.5<z<4.5$ C IV ABSORBERS WITH THOUSANDS OF SYSTEMS
}

\author{
Kathy L. Cooksey ${ }^{1,6}$, Melodie M. KaO ${ }^{2}$, Robert A. Simcoe ${ }^{3}$, John M. O’Meara ${ }^{4}$, and J. Xavier Prochaska ${ }^{5}$ \\ ${ }^{1}$ MIT Kavli Institute for Astrophysics \& Space Research, 77 Massachusetts Avenue, 37-685 Cambridge, MA 02139, USA; kcooksey@ space.mit.edu \\ ${ }^{2}$ Caltech, MC 249-17, 1200 East California Boulevard, Pasadena, CA 91125, USA; mkao@caltech.edu \\ ${ }^{3}$ Department of Physics, MIT, 77 Massachusetts Avenue, 37-664D, Cambridge, MA 02139, USA; simcoe@ space.mit.edu \\ ${ }^{4}$ Department of Chemistry and Physics, Saint Michael's College, One Winooski Park, Colchester, VT 05439, USA; jomeara@ smcvt.edu \\ ${ }^{5}$ Department of Astronomy \& UCO/Lick Observatory, University of California, 1156 High Street, Santa Cruz, CA 95064, USA; xavier@ucolick.org \\ Received 2012 April 11; accepted 2012 November 21; published 2013 January 4
}

\begin{abstract}
We have vastly increased the C IV statistics at intermediate redshift by surveying the thousands of quasars in the Sloan Digital Sky Survey (SDSS) Data-Release 7. We visually verified over 16,000 C IV systems with $1.46<z<4.55$-a sample size that renders Poisson error negligible. Detailed Monte Carlo simulations show that we are approximately $50 \%$ complete down to rest equivalent widths $W_{\mathrm{r}} \approx 0.6 \AA$. We analyzed the sample as a whole and in 10 small redshift bins with approximately 1500 doublets each. The equivalent width frequency distributions $f\left(W_{\mathrm{r}}\right)$ were well modeled by an exponential, with little evolution in shape. In contrast with previous studies that modeled the frequency distribution as a single power law, the fitted exponential gives a finite mass density for the C IV ions. The comoving line density $d \mathcal{N}_{\mathrm{C} \text { IV }} / d X$ evolved smoothly with redshift, increasing by a factor of $2.37 \pm 0.09$ from $z=4.55-1.96$, then plateauing at $d \mathcal{N}_{\mathrm{C} \text { Iv }} / d X \approx 0.34$ for $z=1.96-1.46$. Comparing our SDSS sample with $z<1$ (ultraviolet) and $z>5$ (infrared) surveys, we see an approximately 10 -fold increase in $d \mathcal{N}_{\text {CIV }} / d X$ over $z \approx 6 \rightarrow 0$, for $W_{\mathrm{r}} \geqslant 0.6 \AA$. This suggests a monotonic and significant increase in the enrichment of gas outside galaxies over the 12 Gyr lifetime of the universe.
\end{abstract}

Key words: galaxies: halos - intergalactic medium - quasars: absorption lines - techniques: spectroscopic

Online-only material: color figures, machine-readable tables

\section{INTRODUCTION}

The study of the large-scale structure of the universe provides top-level constraints on models of galaxy evolution. Heavy elements are produced in the stars of galaxies. A variety of feedback processes move these metals from the sites of production into the intergalactic medium (IGM), enriching the material for future generations of stars. The cosmic enrichment cycle generically refers to the movement of gas from inside galaxies to the IGM and, possibly, back again (perhaps many times). The amount of heavy elements, the number of ionizing photons from galaxies and quasars, and the spatial distribution of material are driven by hierarchical structure formation and galactic processes such as star formation and feedback. Spectroscopic surveys of quasars yield a random sample of intervening absorbing gas clouds that can be used to constrain the ongoing and summative enrichment processes in the universe.

C IV $\lambda \lambda 1548,1550$ doublets are important tracers of the IGM and its evolution from $z \approx 6$ to today (Steidel 1990; Barlow \& Tytler 1998; Ellison et al. 2000; Songaila 2001; Boksenberg et al. 2003; Schaye et al. 2003; Scannapieco et al. 2006; Danforth \& Shull 2008; Ryan-Weber et al. 2009; Becker et al. 2009; Cooksey et al. 2010; D'Odorico et al. 2010; Simcoe et al. 2011). This transition has been well studied at $1.5 \lesssim z \lesssim 5.5$ for the following reasons. First, it is a strong transition of a common metal. Second, it is observable outside the Ly $\alpha$ forest, where it becomes easier to identify. Third, it redshifts into optical passbands at $z=1.5$. Lastly, it is a resonant doublet, which gives it distinctive characteristics that enable surveys to be largely automated.

\footnotetext{
${ }^{6}$ NSF Astronomy, and Astrophysics Postdoctoral Fellow.
}

Observations of $\mathrm{C}$ IV doublets constrain the cumulative effect of the cosmic enrichment cycle. More specifically, the number and strength of CIV absorbers are affected by the amount of carbon produced by all previous generations of stars; the spatial distribution of the element, driven by feedback processes; and the total ionizing radiation available to maintain the triply ionized transition.

The intermediate-redshift C IV studies have traditionally found that the doublets follow a power law in the column density distribution function, with a slope of $\alpha \approx-1.8$, throughout the redshift range (Songaila 2001; Boksenberg et al. 2003; Scannapieco et al. 2006; D'Odorico et al. 2010). The earlier studies also measured a roughly constant $\mathrm{C}$ IV mass density for $2<z<4.5$ and $12 \lesssim \log N_{\mathrm{CIV}} \lesssim 15$ absorbers (Songaila 2001, 2005; Boksenberg et al. 2003). Improved observations, pushing to bluer wavelengths (and hence lower redshift), have shown that $\Omega_{\mathrm{C} \text { IV }}$, the C IV mass density relative to the critical density, actually increases smoothly from $z=4 \rightarrow 1.5$ (D'Odorico et al. 2010) and maps well onto the, $z<1$ values, measured with Hubble Space Telescope (HST) ultraviolet spectra (Cooksey et al. 2010). Thus, $\Omega_{\mathrm{CIV}}$ increases by, approximately, a factor of four over $z \approx 3 \rightarrow 0$ while $d \mathcal{N}_{\mathrm{C} \text { IV }} / d X$, the comoving C IV line density, increases by a factor of two, roughly.

Early infrared spectroscopy, which probes the $z \gtrsim 5$ universe, first resulted in a continuation of the roughly constant $\Omega_{\text {Civ }}$ out to $z \approx 6$ (Ryan-Weber et al. 2006; Simcoe 2006). However, with limited sightlines, these studies were highly susceptible to cosmic variance, as shown by later IR surveys, which reported that $\Omega_{\text {CIV }}$ actually dropped at $z \approx 6$ (Ryan-Weber et al. 2009; Becker et al. 2009). The latest and largest IR survey showed that $\Omega_{\text {CIV }}$ drops by approximately a factor of four over $z \approx 4 \rightarrow 6$ (Simcoe et al. 2011), and high-redshift quasars are continuing 
to be observed with the new Folded-port InfraRed Echellete (FIRE) on the Magellan/Baade Telescope (Simcoe et al. 2010). In addition, Simcoe et al. (2011) found that all previous highredshift measurements overestimated $\Omega_{\mathrm{C}_{\text {IV }}}$ by $\approx 30 \%$, since their lower-resolution IR spectra led to the lower-redshift Mg II $\lambda \lambda 2796,2803$ being misidentified as a strong, highredshift $\mathrm{C}$ IV system.

The recent low (UV) and high (IR) redshift publications (Cooksey et al. 2010; Simcoe et al. 2011) have led us to assess the state of the intermediate (optical) redshift field (e.g., Songaila 2001; Boksenberg et al. 2003; Scannapieco et al. 2006; D'Odorico et al. 2010). The various studies have disparities in their definition of an absorber, completeness corrections, sensitivity limits, and/or adopted cosmology. Ideally, there should be a large, uniformly constructed, $0<z<6$ sample in order to evaluate the evolution of the C IV absorbers. We aim to produce an intermediate-redshift catalog that is fairly comparable to the recent $z<1$ and $z>5$ catalogs.

For our $1.5 \lesssim z \lesssim 4.5$ survey, we use more than 26,000 quasar spectra in the Sloan Digital Sky Survey (SDSS; York et al. 2000) Data-Release 7 (DR7) database (Abazajian et al. 2009; Schneider et al. 2010), thus making this study the largest C IV survey-both in path length and in number of absorbers-to date. Others have mined SDSS quasar spectra for $\mathrm{H}_{\mathrm{I}}$ (e.g., Prochaska et al. 2005; Pieri et al. 2010b), Mg II (e.g., Nestor et al. 2005; Prochter et al. 2006), Ca II $\lambda \lambda 3934,3969$ (e.g., Wild et al. 2006; Cherinka \& Schulte-Ladbeck 2011), and O VI $\lambda, \lambda 1031,1037$ (e.g., Frank et al. 2010; Pieri et al. 2010a). However, this is the first time that anyone has systematically searched for C IV, likely because it is more difficult and labor intensive, due to the increased amount of blending. This is the first in a series of papers on metals in SDSS quasar spectra, where we will assemble and analyze self-consistent catalogs of Si IV $\lambda \lambda 1393,1402$, Mg II, and Ca II absorbers. These species are commonly studied in quasar absorption-line spectroscopy and typically trace the gas closest to galaxies.

We explain how we construct our C IV sample in Section 2 and our completeness corrections in Section 3. The main results are detailed in Section 4, and the discussion and summary are in Sections 5 and 6. We adopt the WMAP5 cosmology: $H_{0}=71.9 \mathrm{~km} \mathrm{~s}^{-1} \mathrm{Mpc}, \Omega_{\mathrm{M}}=0.258$, and $\Omega_{\Lambda}=0.742$ (Komatsu et al. 2009).

\section{CONSTRUCTING THE C IV SAMPLE}

\subsection{SDSS Data-Release 7 Quasars}

We began our survey with the 105,783 sightlines in the SDSS DR7 QSO catalog Schneider et al. (2010; also see Richards et al. 2002 for the SDSS quasar selection). SDSS spectra have wavelength coverage $3820 \AA \leqslant \lambda \leqslant 9200 \AA$ and resolution varying from $R=1850$ to 2200 (or $162 \mathrm{~km} \mathrm{~s}^{-1}$ to $136 \mathrm{~km} \mathrm{~s}^{-1}$ ), and the reduced spectra are binned to a log-linear scale of $69 \mathrm{~km} \mathrm{~s}^{-1} \mathrm{pix}^{-1}$. We immediately excluded the 6214 broadabsorption line (BAL) QSOs detailed in Shen et al. (2011).

We limited the sightlines to those with $z_{\mathrm{QSO}} \geqslant 1.7$ and median signal-to-noise ratio $\langle\mathrm{S} / \mathrm{N}\rangle \geqslant 4 \mathrm{pixel}^{-1}$ in the wavelength range sensitive to $\mathrm{C}$ IV absorbers (see Figure 1). This range depended on the quasar redshift and the SDSS wavelength coverage.

Previous, smaller surveys for C IV doublets have searched from the Ly $\alpha \lambda 1215$ to C IV emission wavelengths. However, we removed a portion of this path length close to the Ly $\alpha$ emission to avoid possible confusion between the C IV doublet and any $\mathrm{O}_{\mathrm{I}} \lambda 1302$, Si II $\lambda 1304$ pair. These latter two have

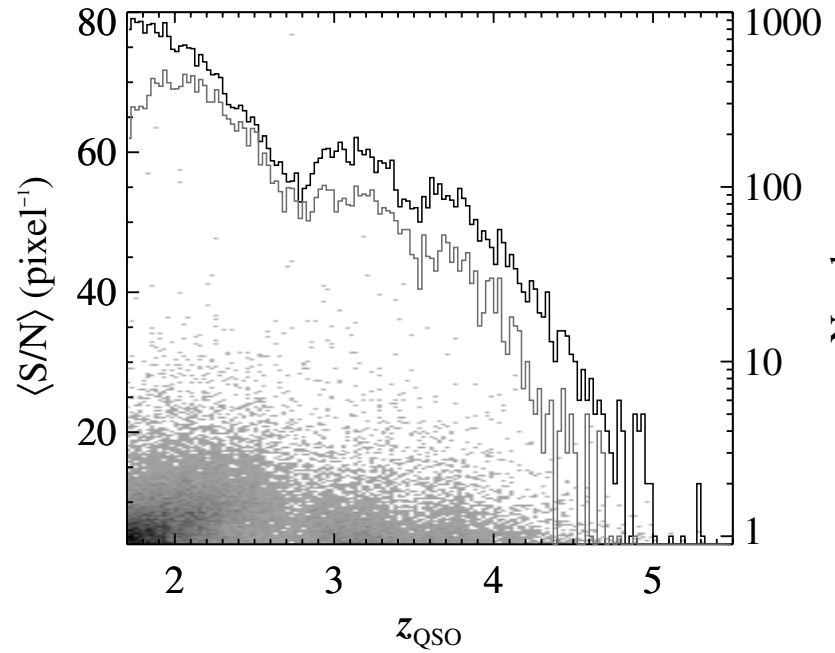

Figure 1. Redshift and $\langle\mathrm{S} / \mathrm{N}\rangle$ distribution of sightlines. Using the left-hand axis, the two-dimensional histogram shows the median signal to noise and $z_{\text {QSO }}$ space of the analyzed 26,030 spectra. The black and gray histograms give the redshift distribution for all spectra and for the 10,861 with confirmed C IV absorbers, respectively (right-hand axis).

a wavelength separation similar enough to the CIV doublet that automated search algorithms naturally bring in a large fraction of false positives. Since we had so much available path length available, we simply excluded a comfortable region around the O I, Si II "forest" in addition to the Ly $\alpha$ forest (i.e., $\lambda_{\mathrm{r}} \geqslant 1310 \AA$ in the rest frame of the quasar). To exclude absorbers intrinsic to the QSO or affected by local ionization, clustering, and/or enrichment, we set the upper wavelength bound to $1548 \AA\left(1+z_{\mathrm{QSO}}\right)\left(1+\delta v_{\mathrm{QSO}} / c\right),{ }^{7}$ where we initially used $\delta v_{\mathrm{QSO}}=-3000 \mathrm{~km} \mathrm{~s}^{-1}$, but for the main analyses, the limit is $\delta v_{\mathrm{QSO}}=-5000 \mathrm{~km} \mathrm{~s}^{-1}$.

After the redshift, $\langle\mathrm{S} / \mathrm{N}\rangle$, and coverage cuts, we had 26,168 sightlines to search (see Table 1). Later, we excluded 138 sightlines as "visual BAL" QSOs for a final count of 26,030 spectra to analyze.

\subsection{Automated Continuum Fitting}

We generated individual quasar continua for all $\approx 26,000$ spectra in the sample using an algorithm that combined principle-component analysis (PCA) fits, low-frequency $\mathrm{b}$-spline correction, and automated outlier pixel and absorptionline exclusion. Since we are interested in C IV lines only, we limited the fit to $\lambda_{\mathrm{r}} \geqslant 1230 \AA$, redward of the Ly $\alpha$ forest. We discuss details of the method below so interested users may replicate it; the codes are also publicly available in the XIDL software library. ${ }^{8}$ Figure 2 shows example fits for eight spectra of varying $\langle\mathrm{S} / \mathrm{N}\rangle$ level.

First, every spectrum was iteratively fit using a 50-vector basis set of PCA "eigenspectra" from the SDSS DR1 quasar sample (Yip et al. 2004). The fit coefficients and their errors were determined using the algorithms of Connolly \& Szalay (1999, Equations (4)-(6)), with spectral pixels weighted by their inverse variance when quantifying the goodness of fit. The formal per-pixel error of the PCA continuum fit was negligible compared to the spectral shot noise.

A pixel exclusion mask was then generated upon each successive fit iteration. The initial mask consisted of the lowest

\footnotetext{
7 Velocity offsets are defined as $\delta v=c\left(z-z_{\text {ref }}\right) /\left(1+z_{\text {ref }}\right)$.

8 See http://www.ucolick.org/ xavier/xidl/.
} 

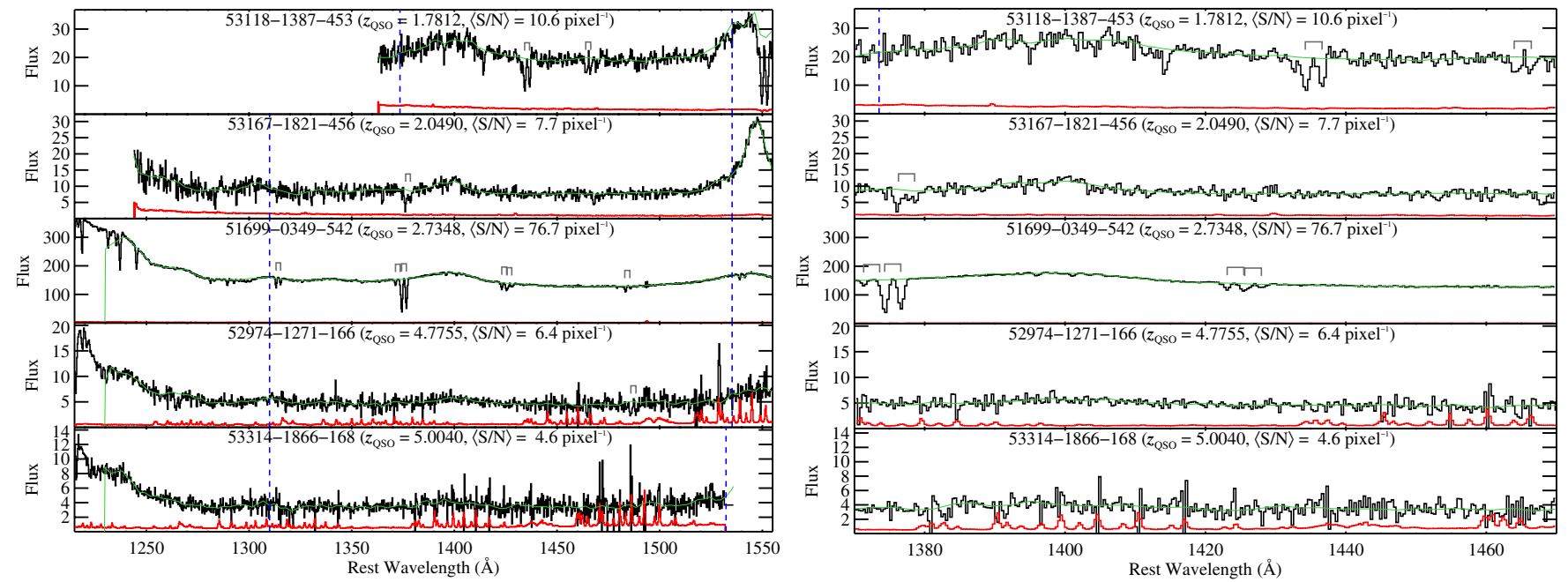

Figure 2. Examples of automated continuum fits. Several QSO spectra (black) and $1 \sigma$ error (red) are shown with their automated continuum fits (green). On the left are the full spectra, and a zoom of the central wavelength range is given on the right. All spectra were fit for $\lambda_{\mathrm{r}} \geqslant 1230 \AA$ with SDSS eigenspectra. For $\langle\mathrm{S} / \mathrm{N}\rangle \geqslant 8$ pixel ${ }^{-1}$ spectra, the eigenfit was adjusted with a b-spline fit to the normalized spectrum. We surveyed the spectra for C IV absorbers between the (blue) vertical lines, which are outside the O I, Si II "forest" and more than $3000 \mathrm{~km} \mathrm{~s}^{-1}$ blueward of the QSO. The gray brackets indicate real C IV doublets from the final sample.

(A color version of this figure is available in the online journal.)

Table 1

Sightline Selection

\begin{tabular}{ll}
\hline \hline Number & \\
\hline 105783 & SDSS DR7 QSO catalog (Schneider et al. 2010) \\
99569 & Excluding 6214 objects in BAL QSO catalog (Shen et al. 2011) \\
48260 & Covering $\left.\max \left[1310 \AA\left(1+z_{\mathrm{QSO}}\right), 3820 \AA\right] \leqslant \lambda \min ^{-1548 \AA}\left(1+z_{\mathrm{QSO}}\right)\left(1+\delta v_{\mathrm{QSO}} / c\right), 9200 \AA\right]^{\mathrm{a}}$ \\
26168 & With $z_{\mathrm{QSO}} \geqslant 1.7$ and $\langle\mathrm{S} / \mathrm{N}\rangle \geqslant 4$ pix $^{-1}$ in above wavelength range ${ }^{\mathrm{b}}$ \\
26030 & Excluding 138 visual BAL QSOs \\
10861 & With confirmed C IV doublets \\
\hline
\end{tabular}

Notes.

a The $1310 \AA$ A limit excludes the contaminating region in the O I, Si II "forest." The fiducial value for $\delta v_{\mathrm{QSO}}=-3000 \mathrm{~km} \mathrm{~s}^{-1}$.

$\mathrm{b}$ The details of these specific sightlines are given in Table 2.

Table 2

Sightline Summary

\begin{tabular}{|c|c|c|c|c|c|c|c|c|c|}
\hline $\begin{array}{l}\text { (1) } \\
\text { QSO ID }\end{array}$ & $\begin{array}{l}(2) \\
\text { R.A. }\end{array}$ & $\begin{array}{c}(3) \\
\text { Decl. }\end{array}$ & $\begin{array}{c}(4) \\
z_{\mathrm{QSO}}\end{array}$ & $\begin{array}{c}(5) \\
\langle\mathrm{S} / \mathrm{N}\rangle \\
\left(\text { pixel }^{-1}\right)\end{array}$ & $\begin{array}{c}(6) \\
f_{\mathrm{BAL}}\end{array}$ & $\begin{array}{c}(7) \\
\Delta X_{\max }\end{array}$ & $\begin{array}{c}(8) \\
\mathcal{N}_{\text {cand }}\end{array}$ & $\begin{array}{c}(9) \\
\mathcal{N}_{\text {C IV }}\end{array}$ & $\begin{array}{c}(10) \\
\delta X_{\mathrm{CIV}}\end{array}$ \\
\hline 52235-0750-082 & 00:00:09.38 & $+13: 56: 18.4$ & 2.2342 & 5.94 & 0 & 1.33 & 0 & 0 & \\
\hline 52203-0685-198 & 00:00:14.82 & $-01: 10: 30.7$ & 1.8877 & 5.16 & 0 & 1.24 & 0 & 0 & \\
\hline 52203-0685-439 & 00:00:15.47 & $+00: 52: 46.8$ & 1.8516 & 8.64 & 0 & 2.16 & 0 & 0 & \\
\hline 54389-2822-315 & 00:00:24.83 & $+24: 57: 03.3$ & 3.2137 & 9.21 & 0 & 1.48 & 0 & 0 & \\
\hline 52203-0685-154 & 00:00:53.17 & $-00: 17: 32.9$ & 2.7571 & 7.55 & 0 & 1.15 & 0 & 0 & \\
\hline 52902-1091-546 & 00:00:57.58 & $+01: 06: 58.6$ & 2.5551 & 11.83 & 0 & 1.78 & 3 & 2 & 0.084 \\
\hline 51791-0387-093 & 00:00:58.22 & $-00: 46: 46.5$ & 1.8973 & 13.27 & 0 & 0.84 & 1 & 1 & 0.037 \\
\hline $52203-0685-134$ & $00: 01: 25.14$ & $+00: 00: 09.4$ & 1.9739 & 5.32 & 0 & 1.52 & 0 & 0 & \\
\hline
\end{tabular}

Notes. Column 1 is the adopted QSO identifier from the spectroscopic modified Julian date, plate, and fiber number. Columns 2 through 4 are from the DR7 QSO catalog (Schneider et al. 2010). Column 5 is the median $\mathrm{S} / \mathrm{N}$ measured in the region searched for $\mathrm{C}$ IV. The binary BAL flag $f_{\mathrm{BAL}}$ in Column 6 indicates which sightlines were considered BALs by at least one author (4) and which were confirmed by the authors as BALs to exclude (8). Column 7 is the maximum comoving path length available in the sightline. Columns 8 and 9 give the number of candidate and confirmed Civ doublets, respectively. Column 10 is the path length blocked by the $\mathcal{N}_{\mathrm{C} \text { IV }}$ doublets in the sightline.

(This table is available in its entirety in a machine-readable form in the online journal. A portion is shown here for guidance regarding its form and content.) 
$30 \%$ of pixels in successive 50 pixel windows, to filter strong absorption features and avoid biasing the continuum low on the critical initial fit. Then, for each subsequent iteration, a fresh mask was constructed that excluded pixels falling either less than $-2 \sigma$ or more than $3 \sigma$ from the working continuum. This mask was further modified to include the neighboring pixels of each deviant pixel below the working continuum and exclude regions with three consecutive pixels above. The latter correspond to systematically errant regions of the fit that should actually be included, so they were unmasked automatically. This cycle was repeated with successive PCA fits until: (1) the mask converged so successive fits did not change; (2) the unmasked flux and continuum fit had median difference less than $0.001 \%$; or (3) 10 iterations passed.

For high-S/N spectra, low-frequency residuals in the PCAnormalized spectra become noticeable and affect our ability to search for weak C IV lines in the best data. We therefore performed a secondary correction to spectra with $\langle\mathrm{S} / \mathrm{N}\rangle \geqslant$ 8 pixel $^{-1}$, re-fitting the PCA-normalized spectrum with an additional third-order b-spline, with breakpoint spacing of 25 pixels (or $1725 \mathrm{~km} \mathrm{~s}^{-1}$ ). As before, we used inverse-variance weighting and clipped outliers using the same thresholds. We refer below to this PCA plus b-spline fit as the "hybrid continuum."

The b-spline systematic error was estimated as the median difference between the b-spline and the PCA-normalized (masked) spectrum in bins matching the breakpoint spacing. This error was typically of the order of $1 \%$ of the shot noise in the data; it exceeded the formal PCA fit error, and we added the two in quadrature to produce a total continuum error.

The sigma clipping methods described above are effective at identifying narrow features, instrumental artifacts, or defects in the spectra. However, they are not always optimal for finding true absorption lines, which are always below the continuum; they can also be kinematically complex and bias the fit. Since we have prior information about the characteristic widths of cosmological absorption, we develop methods in Section 2.3 to machine-identify candidate absorption lines with these characteristics for the actual survey. We ran each PCA-normalized spectrum through a single pass of the absorption-line finder, using the resulting catalogs to generate a master absorption mask for final hybrid-continuum fitting.

The full procedure for automated feature finding is described in Section 2.3; briefly, we convolve both the data and error arrays with the instrumental response profile, and search for absorption features with $\mathrm{S} / \mathrm{N} \geqslant 3.5$ per resolution element. For $\langle\mathrm{S} / \mathrm{N}\rangle \leqslant 16$ pixel $^{-1}$ spectra, we masked out all such features; in high-S/N cases, we also enforced that the unconvolved spectral pixels at the line center must deviate from the continuum by $>5 \sigma$. Pixels falling within $\pm 600 \mathrm{~km} \mathrm{~s}^{-1}$ of such features were added to the exclusion set. The mask consisting of automatically identified absorption lines was fed into the hybrid-continuum fit as a static mask.

This procedure produced excellent continuum fits for the vast majority of spectra. When it failed, the most common reasons were poorly measured $z_{\mathrm{QSO}}$, strong intrinsic absorption, BALs, and foreground emission-line galaxy spectra superimposed on the quasar spectra. Instead of fixing these cases interactively, which would compromise our objective methodology and continuum error estimates, we chose to leave them in the sample and let the effects be accounted in our automated completeness and contamination tests (see Section 3).

\subsection{Automated Candidate Selection}

We limited our candidate search to C IV doublets where two automatically detected lines were within $\pm 150 \mathrm{~km} \mathrm{~s}^{-1}$ of the characteristic velocity separation $\delta v_{\text {CIV }}=498 \mathrm{~km} \mathrm{~s}^{-1}$. The large uncertainty cut allowed heavily blended lines into our candidate list, but it also made the $\mathrm{Mg}$ II doublet our largest contaminant since $\delta v_{\mathrm{Mg} \text { II }}=767 \mathrm{~km} \mathrm{~s}^{-1}$.

Our automated feature-finding algorithm is based on Prochter et al. (2006). The hybrid-normalized flux and error arrays were convolved with a Gaussian kernel with FWHM equivalent to a resolution element of the SDSS spectrograph, i.e., $\sigma_{\mathrm{G}}=1$ pixel. The error array included the continuum fit error. The regions where the convolved signal-to-noise $(\mathrm{S} / \mathrm{N})_{\text {conv }}$ is greater than or equal to 3.5 per resolution element were identified as absorption features and saved to the sightline's line list. We masked out the few pixels $(\approx 5 \AA)$ around the strong skylines at $5579 \AA$ and $6302 \AA$.

Candidate $\mathrm{C}$ IV doublets were compiled by pairing automatically detected lines with the characteristic velocity separation $\delta v_{\text {CIV }} \pm 150 \mathrm{~km} \mathrm{~s}^{-1}$ and by identifying isolated, automatically detected lines that were broad enough to be a candidate doublet by themselves. As discussed in Section 2.1, the search region is set so that the C IV candidate is redward of the O I, Si II "forest" $\left(\lambda_{\mathrm{r}} \geqslant 1310 \AA\right)$ and blueward of the QSO by $3000 \mathrm{~km} \mathrm{~s}^{-1}$. For any unpaired line, we re-ran the automated feature finder with a $2.5 \sigma_{\text {conv }}$ cutoff and searched for a partner candidate 1550 line.

To identify isolated, broad lines that should be candidate C IV doublets, we used the automated procedure for measuring wavelength bounds (described in Section 2.4) to find lines with widths $\delta v_{\text {lin }} \geqslant 1.5 \delta v_{\text {C IV }}$.

Ultimately, we identified 29,789 candidates, 6346 of which were from the broad-line search.

\subsection{Measuring Absorber Properties}

The redshift, equivalent width, and column density of a line depend on the definition of its wavelength bounds. The bounds were automatically defined by where the convolved $\mathrm{S} / \mathrm{N}$ array stopped decreasing from the perspective of the automatically detected centroid, and the bounds were not allowed to exceed the midpoint between themselves and neighboring lines. Thus, for $\mathrm{C}$ IV lines, the inner wavelength bounds were not allowed to exceed the midpoint of the doublet.

The redshift, equivalent width, and column density errors included the estimated error due to the hybrid continuum. Redshifts were measured from the flux-weighted centroids. Equivalent widths were measured by simply summing the absorbed flux within the bounds.

We used the apparent optical depth method (AODM; Savage $\&$ Sembach 1991) to estimate column densities. Systems that are saturated (discussed below) formally have column densities that are lower limits (binary flag $f_{N}=2$ ). Column density measurements that are less than $3 \sigma_{N}$ are flagged as upper limits $\left(f_{N}=4\right)$.

With doublets, we have two measurements of the column density, and we use both to set $N_{\text {CIV }}$, as done in Cooksey et al. (2010). For doublets where both lines are measurements (not limits), $N_{\mathrm{C} \text { IV }}$ is the inverse-variance weighted mean of the two values. If one line is a measurement, $N_{\mathrm{C}_{\text {IV }}}$ is set to its value. The column density limit is set to the inverse-variance weighted mean when both doublet limits are the same kind. When the doublet limits bracket a range, $N_{\mathrm{C} \text { IV }}$ is set to the unweighted average with an error that reflects the range $\left(f_{N}=8\right)$. In the 
Table 3

Civ System Summary

\begin{tabular}{|c|c|c|c|c|c|c|c|}
\hline $\begin{array}{l}\text { (1) } \\
\text { QSO ID }\end{array}$ & $\begin{array}{c}(2) \\
z_{\mathrm{QSO}}\end{array}$ & $\begin{array}{c}(3) \\
z_{1548}\end{array}$ & $\begin{array}{c}(4) \\
W_{\mathrm{r}, 1548} \\
(\AA)\end{array}$ & $\begin{array}{c}(5) \\
W_{\mathrm{r}, 1550} \\
(\AA)\end{array}$ & $\begin{array}{c}(6) \\
C\left(W_{\mathrm{r}, 1548}\right)\end{array}$ & $\begin{array}{c}(7) \\
\log N_{\mathrm{C}_{\text {IV }}} \\
\left(\log \left(\mathrm{cm}^{-2}\right)\right)\end{array}$ & $\begin{array}{l}(8) \\
f_{N}\end{array}$ \\
\hline 52143-0650-199 & 1.8449 & 1.52755 & $0.567 \pm 0.147$ & $0.981 \pm 0.162$ & $0.562 \pm 0.206$ & $>14.30 \pm 0.14$ & 3 \\
\hline \multirow[t]{2}{*}{$52143-0650-178$} & 2.6404 & 2.23461 & $0.425 \pm 0.059$ & $0.303 \pm 0.058$ & $0.363 \pm 0.092$ & $>14.10 \pm 0.06$ & 3 \\
\hline & & 2.43947 & $1.050 \pm 0.078$ & $0.653 \pm 0.072$ & $0.860 \pm 0.023$ & $>14.53 \pm 0.03$ & 3 \\
\hline \multirow[t]{2}{*}{$52902-1091-546$} & 2.5551 & 2.34348 & $0.734 \pm 0.202$ & $0.898 \pm 0.198$ & $0.713 \pm 0.192$ & $>14.75 \pm 0.14$ & 3 \\
\hline & & 2.42902 & $1.053 \pm 0.191$ & $0.733 \pm 0.196$ & $0.860 \pm 0.066$ & $? 14.63 \pm 0.20$ & 7 \\
\hline 51791-0387-093 & 1.8973 & 1.77015 & $0.388 \pm 0.097$ & $0.479 \pm 0.098$ & $0.305 \pm 0.132$ & $>14.01 \pm 0.13$ & 3 \\
\hline $52235-0750-550$ & 2.6383 & 2.16695 & $0.581 \pm 0.123$ & $0.594 \pm 0.127$ & $0.576 \pm 0.161$ & $>14.23 \pm 0.09$ & 3 \\
\hline $54389-2822-423$ & 2.7668 & 2.36223 & $0.738 \pm 0.087$ & $0.332 \pm 0.088$ & $0.716 \pm 0.073$ & $14.34 \pm 0.06$ & 1 \\
\hline $54327-2630-423$ & 2.7251 & 2.23946 & $0.489 \pm 0.100$ & $0.390 \pm 0.102$ & $0.458 \pm 0.151$ & $>14.16 \pm 0.11$ & 3 \\
\hline \multirow[t]{5}{*}{$54452-2824-554$} & 2.5566 & 2.08349 & $0.837 \pm 0.040$ & $1.050 \pm 0.042$ & $0.781 \pm 0.025$ & $>14.55 \pm 0.02$ & 3 \\
\hline & & 2.08788 & $0.971 \pm 0.039$ & $0.398 \pm 0.039$ & $0.836 \pm 0.014$ & $14.46 \pm 0.02$ & 1 \\
\hline & & 2.14618 & $0.769 \pm 0.041$ & $0.462 \pm 0.037$ & $0.738 \pm 0.030$ & $>14.39 \pm 0.03$ & 3 \\
\hline & & 2.21361 & $0.381 \pm 0.039$ & $0.254 \pm 0.041$ & $0.294 \pm 0.059$ & $>14.00 \pm 0.05$ & 3 \\
\hline & & 2.38185 & $0.395 \pm 0.039$ & $0.284 \pm 0.039$ & $0.317 \pm 0.062$ & $>14.05 \pm 0.05$ & 3 \\
\hline $52143-0650-561$ & 1.7593 & 1.63718 & $0.393 \pm 0.049$ & $0.185 \pm 0.053$ & $0.314 \pm 0.075$ & $13.99 \pm 0.07$ & 1 \\
\hline $52991-1489-581$ & 1.8536 & 1.69764 & $1.013 \pm 0.090$ & $0.812 \pm 0.088$ & $0.849 \pm 0.030$ & $>14.55 \pm 0.04$ & 3 \\
\hline \multirow[t]{2}{*}{$52203-0685-567$} & 2.0921 & 1.70301 & $1.165 \pm 0.186$ & $0.669 \pm 0.180$ & $0.877 \pm 0.038$ & $14.57 \pm 0.08$ & 1 \\
\hline & & 1.75102 & $0.875 \pm 0.121$ & $0.827 \pm 0.122$ & $0.799 \pm 0.070$ & $>14.54 \pm 0.07$ & 3 \\
\hline
\end{tabular}

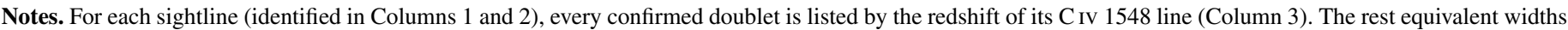

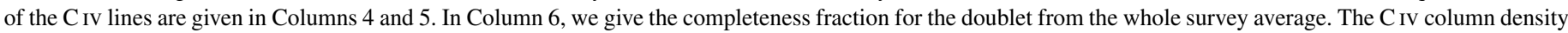

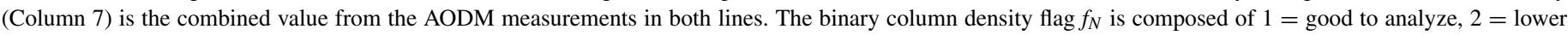
limit, $4=$ upper limit, $8=$ unweighted average, and 16= default to line value with greater significance (Column 8).

(This table is available in its entirety in a machine-readable form in the online journal. A portion is shown here for guidance regarding its form and content.)

remaining cases, $N_{\mathrm{C}_{\text {IV }}}$ is set to the better measurement/limit and flagged $f_{N}=16$.

The AODM systematically overestimates the true column densities in low-S/N spectra (Fox et al. 2005). On the other hand, most doublets that can be detected in the low-resolution SDSS spectra are saturated, as evidenced by the doublet ratios of near unity; therefore, the AOD column densities would formally be lower limits. We define the doublet to be unsaturated when the equivalent width ratio $W_{\mathrm{r}, 1548} / W_{\mathrm{r}, 1550}>2-\sigma_{\mathrm{R}}$, where $\sigma_{\mathrm{R}}$ is the ratio error due to the uncertainties in the measured equivalent widths. All other doublets are flagged as saturated with column densities that are lower limits; by this criterion, approximately $84 \%$ of the final catalog are saturated (see Table 3).

\subsection{Interactive Doublet Verification}

The candidate $\mathrm{C}$ IV selection relied solely on the characteristic wavelength separation of the doublet. However, true, unblended C IV doublets have a well-defined doublet ratio $W_{\mathrm{r}, 1548} / W_{\mathrm{r}, 1550}$ in the range of one to two in the saturated and unsaturated regimes, respectively. The resolved profiles of the doublet lines are very similar in the absence of blending. Most C IV absorbers are associated with $\operatorname{Ly} \alpha$ absorption, though "naked" systems may exist (Schaye et al. 2007). There are frequently other metal lines associated with the C IV absorption, such as the Si IV and/or Mg II doublets. In addition, outside of the Ly $\alpha$ (and O I, $\mathrm{Si}$ II) forest, line confusion, and blending are less severe, and the $\mathrm{C}$ IV doublet is one of the more common metal lines.

We developed a graphical user interface to present all this information for each system to assist us in rating the 29,789 candidates. We leveraged our experience with C IV absorption systems and the varying amounts of information available, depending on redshift, to assign each candidate a rating:
0 . definitely false-either can definitively identify the lines as other metal lines or as spurious bad pixels masquerading as absorption;

1. likely false - though cannot name alternate metal-line identification, better data would probably confirm this as not C IV absorption;

2. likely true- - though sparse supporting evidence (e.g., associated lines), better data would probably confirm this as C IV absorption; or

3. definitely real-associated with other lines and/or shows clear correlations in line profiles.

A single author was trusted to accurately assign ratings of 0 or 3 ; these doublets were not viewed again. The more ambiguous cases (ratings 1 and 2) were reviewed by at least one additional author until consensus was achieved. We grouped the doublets into systems with $\delta v_{\mathrm{abs}}<250 \mathrm{~km} \mathrm{~s}^{-1}$.

Though we excluded the BAL QSOs from Shen et al. (2011), we found several sightlines with strong, self-blended, highly blueshifted C IV absorbers. Since we focused on the intergalactic absorption systems, we excluded these 138 sightlines from further analysis and labeled them "visual BAL" QSOs in Table 2 (binary flag $f_{\mathrm{BAL}}=12$ ). In addition, the extremely strong absorption lines in these spectra corrupted the automated continuum fitting algorithm.

The final catalog includes all doublets with rating $\geqslant 2$. From the initial 29,789 candidates, 743 were in sightlines excluded as visual BAL QSOs. In the remaining 29,046 candidates, we found 16,459 real Civ doublets (see Table 3). A sample of doublets are shown in Figure 3. Ultimately, we analyzed the 14,772 with $\delta v_{\mathrm{QSO}}<-5000 \mathrm{~km} \mathrm{~s}^{-1}$ (see Section 4.1).

We assessed the effects of blending and bad continua by inspecting 4500 doublets chosen at random. We flagged doublets where the 1548,1550 , or both lines were blended or otherwise 


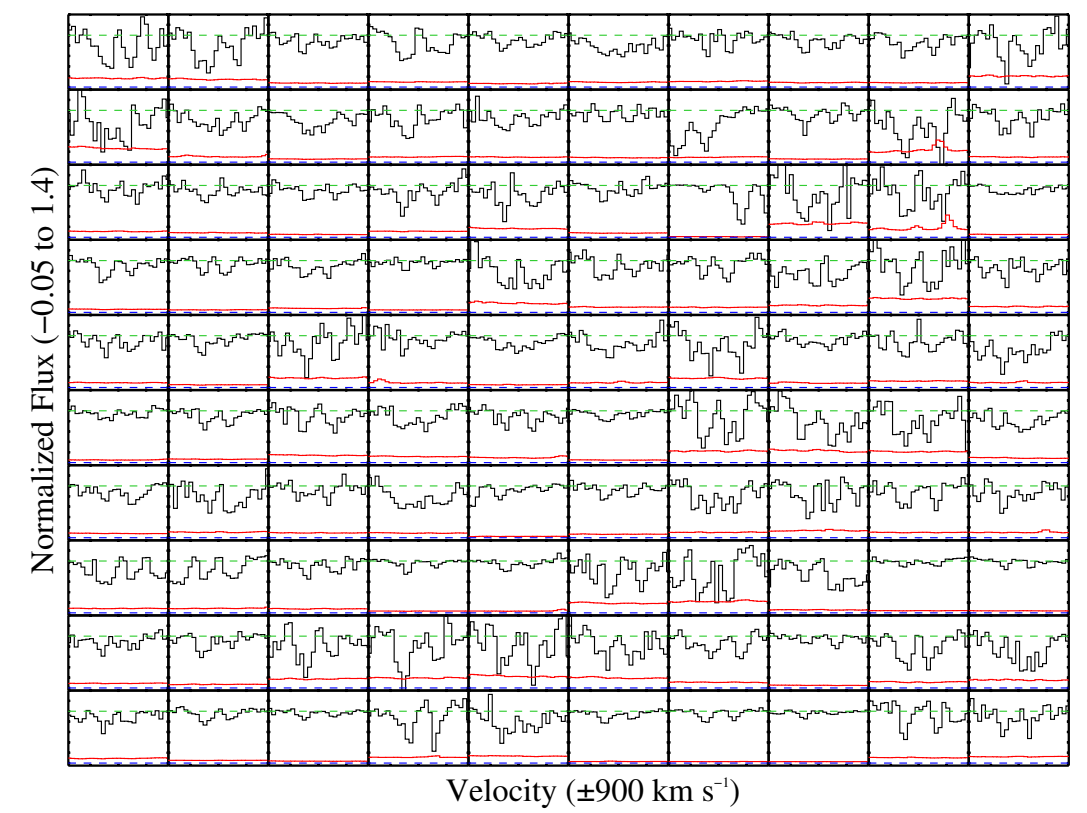

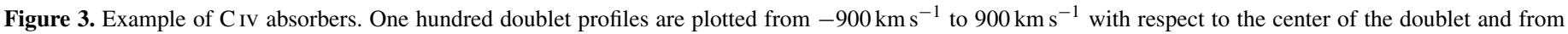

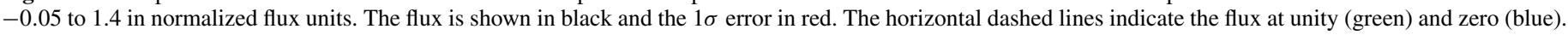
(A color version of this figure is available in the online journal.)

problematic (e.g., the bounds were unrealistic) and where the continua ought to be adjusted locally (e.g., due to strong absorption or neighboring emission lines). The majority of our analysis depended on $W_{\mathrm{r}, 1548}$, so it mattered most what fraction of the 1548 lines were troublesome.

Over $90 \%$ of the random sample had "perfect" treatment of the 1548 line, and over $95 \%$ had acceptable continuum fits. The problematic doublets showed some redshift dependence due to the confused nature in the skyline region $\lambda_{\text {obs }} \gtrsim 7500 \AA$ or $z_{1548} \gtrsim 3.8$, where the fraction of problematic 1548 lines jumped from $\approx 10 \%$ to $\approx 20 \%$. The stronger lines $\left(W_{\mathrm{r}, 1548} \gtrsim\right.$ $2 \AA$ ) were more often blended. These systems tended to be self-blended and would generally be problematic to surveys using boxcar summation for equivalent widths, such as ours. To maintain the largely automated, and hence objective and repeatable, nature of the survey, we decided to accept the imperfections and assess the strength of our results in light of them.

\section{COMPLETENESS TESTS}

We test our survey completeness by generating a library of synthetic profiles, randomly distributing them in a subset of the sightlines, and tracking which simulated doublets were recovered. The goal was to populate a grid of doublet redshifts and rest equivalent widths with the fraction of recovered and accepted simulated doublets in each grid cell. We aimed to sample the full distribution of completeness limits so that we could leverage the large number of sightlines; for example, two spectra with $50 \%$ completeness in one $\left(W_{\mathrm{r}}, z_{1548}\right)$ cell are equivalent to one sightline with $100 \%$ completeness for the same parameters (Matejek \& Simcoe 2012). In this section, we detail how we generated synthetic profiles, input and recover them, and measure the completeness, in addition to discussing biases.

\subsection{Simulated Doublets}

We parameterized the simulated doublets to produce absorption lines that look like the diversity of profiles observed in the
SDSS spectra. We created a Voigt profile library with a uniform distribution of equivalent widths, since we aimed to generate uniform errors on detection completeness as a function of $W_{\mathrm{r}, 1548}$. Each component had a column density that was sampled linearly from $11<\log N_{\mathrm{C}_{\text {IV }}}<14$ and a Doppler parameter randomly selected in the range $5 \mathrm{~km} \mathrm{~s}^{-1}<b<40 \mathrm{~km} \mathrm{~s}^{-1}$. For each system, one primary component was generated, and a random draw from a Poisson distribution, with mean of $\mu_{\text {comp }}^{\text {Poiss }}$, determined the number of additional components for the system. The velocity offset for each component was drawn from a Gaussian distribution with standard deviation $\sigma_{\delta v}^{\text {Gauss }}$, clipped to keep all components within the maximum offset $\delta v_{\max }$ relative to the system redshift.

The bulk of our profiles were generated with $\mu_{\text {comp }}^{\text {Poiss }}=4$ or $7, \sigma_{\delta v}^{\text {Gauss }}=100 \mathrm{~km} \mathrm{~s}^{-1}$, and $\delta v_{\max }=300 \mathrm{~km} \mathrm{~s}^{-1}$, which were roughly based on the component properties measured by Boksenberg et al. (2003). However, to reproduce the diversity of profiles found in the SDSS data, we had to modify the parameters (e.g., larger $\mu_{\text {comp }}^{\text {Poiss }}$ and $\delta v_{\max }$ ), and we limited the alternate parameters to the larger equivalent width regime (e.g., $W_{\mathrm{r}} \geqslant 1.2 \AA$ ), where Boksenberg et al. (2003) did not have many systems.

We generated over $10^{5}$ Voigt profiles. Then we randomly selected or duplicated (in extreme equivalent width regimes) profiles to uniformly sample $0.05 \AA<W_{\mathrm{r}, 1548}<3.25 \AA$ in bins of $0.05 \AA$, resulting in a library of 32,000 profiles.

\subsection{Monte Carlo Procedures}

We have two Monte Carlo completeness tests, referred to as basic and user. The basic test included all of the automated procedures, from continuum fitting to candidate selection. It was applied to the largest fraction of sightlines $(\approx 30 \%)$. We tested the user bias by visually inspecting the candidates in $\approx 6 \%$ of the sightlines. The subsamples were set by requiring at least one sightline in each bin of $\Delta z_{\mathrm{QSO}}=0.25$ for $z_{\mathrm{QSO}} \geqslant 1.7$ and $\Delta\langle\mathrm{S} / \mathrm{N}\rangle=0.25 \mathrm{pixel}^{-1}$ for $\langle\mathrm{S} / \mathrm{N}\rangle \geqslant 4 \mathrm{pixel}^{-1}$. For the basic test, with more sampled sightlines, we continued sampling from 
the sightlines in these $\Delta z_{\mathrm{QSO}}$ and $\Delta\langle\mathrm{S} / \mathrm{N}\rangle$ bins, so that the bins with more sightlines had more tested for completeness. Extra high-redshift sightlines were included in the user test in increase the statistics.

We chose to input the simulated doublets into the actual spectra in order to sample realistic data. We "cleaned" each spectrum by removing a random $30 \%$ of the automatically detected absorption features. The flux between the wavelength bounds (see Section 2.4) was replaced by continuum with a scatter drawn from neighboring pixels, and the new error reflected those same pixels. By leaving the remaining absorption features in, we were able to measure the effects of blending and of absorption lines on the automated continuum fit and linefinding algorithm.

We set a fiducial absorber redshift density $d \mathcal{N}_{\text {C IV }} / d z=5$ to determine how many simulated doublets to input into the cleaned spectrum. This translated to typically injecting one to three doublets per loop, and the test iterated until at least 1000 simulated doublets were input per sightline. We did not change the error array when injecting profiles, which slightly but systematically lowered the $\mathrm{S} / \mathrm{N}$ of the simulated doublets.

For every sightline in the basic test, a random sample of profiles were drawn from our library and assigned redshifts to fall within the limits of the current sightline. The spectrum was "cleaned" to start and every 50th loop thereafter. We injected one to three simulated doublets, fit the hybrid continuum (see Section 2.2), and ran the automated candidate identification algorithm (see Section 2.3). Any injected profile was flagged as recovered if the automatically identified candidate bounds spanned the observed wavelength of the simulated system. For any profile that was not recovered, we measured the fluxweighted redshift, equivalent width, and AOD column density at the expected location. The input and recovered information was stored for later processing (see Section 3.3).

The user completeness test served a dual purpose. It tested the effects of human bias (e.g., we were less likely to accept real doublets at high redshift due to poor sky subtraction at $\gtrsim 7000 \AA$ ) and our accepted false-positive rate (i.e., how often we rated non-C IV lines as $C_{\text {IV }}$ absorption). The steps were largely the same for one sightline in the user test as in the basic, but we "cleaned" the profile every iteration and an author rated the automatically detected candidates. However, we modified the simulated doublets to have an unphysical characteristic wavelength separation of $4.8 \AA$ or $924 \mathrm{~km} \mathrm{~s}^{-1}$ so that any candidate was most likely either simulated or spurious. The fake $\lambda \lambda 1547,1552$ profile library was truncated to $W_{\mathrm{r}, 1547} \leqslant 1 \AA$. We did not simulate other absorption lines because, fundamentally, we are conducting a blind C IV survey.

\subsection{Completeness Correction and Unblocked Comoving Path Length}

We combined the completeness tests in bins of redshift and onto grids of equivalent width so that we correct any detected absorber based on its completeness fraction. The estimated completeness fraction for a given grid point $\left(z_{\mathrm{g}}, W_{\mathrm{g}}\right)$ is simply $\mathcal{N}_{\text {accept }} / \mathcal{N}_{\text {input }}$, the fraction of input C IV doublets that are recovered automatically and accepted by the user, in the given grid cell. The two completeness tests separately measured the recovering and accepting effects so that the final completeness fraction is estimated by

$$
C\left(z_{\mathrm{g}}, W_{\mathrm{g}}\right)=\frac{\mathcal{N}_{\text {rec }}\left(z_{\mathrm{g}}, W_{\mathrm{g}}\right)}{\mathcal{N}_{\text {input }}\left(z_{\mathrm{g}}, W_{\mathrm{g}}\right)} \frac{\mathcal{N}_{\text {accept }}\left(z_{\mathrm{g}}, W_{\mathrm{g}}\right)}{\mathcal{N}_{\text {rec }}\left(z_{\mathrm{g}}, W_{\mathrm{g}}\right)},
$$

where the right-hand product can be thought of as $C_{\text {basic }} C_{\text {user }}$. The completeness grid $C\left(z_{\mathrm{g}}, W_{\mathrm{g}}\right)$ collapses to a curve $C\left(W_{\mathrm{g}}\right)$ in a fixed redshift bin. The basic completeness uncertainty $\sigma_{C_{\text {basic }}}$ is estimated by the Wilson score interval for a binomial distribution (Wilson 1927). This confidence interval estimator is well behaved for small $\mathcal{N}_{\text {input }}$ and/or for extreme completeness fractions.

The statistics on the user completeness were naturally smaller than for the basic, so we fit $C_{\text {user }}\left(W_{\mathrm{r}}\right)$ with the following model:

$$
C_{\mathrm{user}}\left(W_{\mathrm{r}}\right)=C_{0}\left(1-e^{\beta\left(W_{\mathrm{r}}-W_{0}\right)}\right)
$$

in two redshift bins. The dividing $z=2.97$ corresponded to the beginning of the skyline region at $\approx 7000 \AA$, with its resulting decrease in $C_{\mathrm{user}}$ due to confusion, and matched the start of the highest redshift bin. The fit uncertainties were estimated from Monte Carlo re-sampling of the equivalent width errors, and the final error on $C\left(W_{\mathrm{r}}\right)$ was propagated from $\sigma_{C_{\text {basic }}}$ and $\sigma_{C_{\text {user }}}$. We extrapolated the fits to larger equivalent widths when we calculated the full completeness fraction. The user completeness test is discussed in detail in Section 3.4 below.

We scaled $C\left(z_{\mathrm{g}}, W_{\mathrm{g}}\right)$ by the fraction of the total path length not obscured by doublets with greater equivalent widths, in the redshift bin. All C IV lines blocked $<2 \%$ of the total survey path length (see Table 2).

In actuality, $\mathcal{N}_{\text {input }}$ and $\mathcal{N}_{\text {rec }}$ contain the information only for the profiles and sightlines actually sampled in the basic completeness test. Any sightline that did not have a measurement for one or more quantities was accounted for with the average of the sightlines actually tested in the requested $\Delta z_{\mathrm{Q} S O}$ and $\Delta\langle\mathrm{S} / \mathrm{N}\rangle$ bin.

The unblocked comoving path length ${ }^{9}$ for each grid cell is calculated by simply multiplying the completeness fraction by the total path length available in each redshift bin:

$$
\begin{gathered}
\Delta X\left(W_{\mathrm{g}}\right)=\Delta X\left(z_{\mathrm{g}}\right) C\left(z_{\mathrm{g}}, W_{\mathrm{g}}\right) \\
\sigma_{\Delta X\left(W_{\mathrm{g}}\right)}^{2}=\Delta X\left(z_{\mathrm{g}}\right)^{2} \sigma_{C(z, W)}^{2} .
\end{gathered}
$$

In Figure 4 , the black curves are the grid values $\Delta X\left(W_{\mathrm{g}}\right)$ and errors.

The unblocked comoving path length to which our survey is sensitive for any given detected equivalent width $W_{\mathrm{i}}$ is interpolated from the grid of $\Delta X\left(W_{\mathrm{g}}\right)$ :

$$
\begin{aligned}
\Delta X\left(W_{\mathrm{i}}\right)= & \operatorname{interpol}\left(\Delta X\left(W_{\mathrm{g}}\right), W_{\mathrm{g}}, W_{\mathrm{i}}\right) \\
\sigma_{\Delta X}^{2}= & \operatorname{interpol}\left(\sigma_{\Delta X\left(W_{\mathrm{g}}\right)}^{2}, W_{\mathrm{g}}, W_{\mathrm{i}}\right) \\
& +\left(\Delta X\left(W_{\mathrm{i}}\right)-\Delta X\left(W_{\mathrm{i}} \pm \sigma_{W_{\mathrm{i}}}\right)\right)^{2} .
\end{aligned}
$$

The error on $\Delta X\left(W_{\mathrm{i}}\right)$ accounts for the error in our completeness correction $\left(\sigma_{\Delta X}\right)$ and for the uncertainty in our equivalent width measurement $\left(\sigma_{W_{\mathrm{i}}}\right)$. We plot each doublet's $\Delta X\left(W_{\mathrm{r}, 1548}\right)$ in gray in Figure 4. Clearly, the uncertainty in equivalent width dominates the error in the completeness correction.

In all redshift bins, the typical completeness fraction reached $50 \%$ by $W_{\mathrm{r}, 1548} \approx 0.6 \AA$, a value we frequently use as the minimum in subsequent analyses.

\subsection{Automated and Interactive Biases}

The completeness curves do not monotonically increase to $100 \%$ at large equivalent widths (see Figure 4). Instead,

\footnotetext{
9 The comoving path length is related to redshift as follows:

$X(z)=2 \sqrt{\Omega_{\mathrm{M}}(1+z)^{3}+\Omega_{\Lambda}} /\left(3 \Omega_{\mathrm{M}}\right)$.
} 


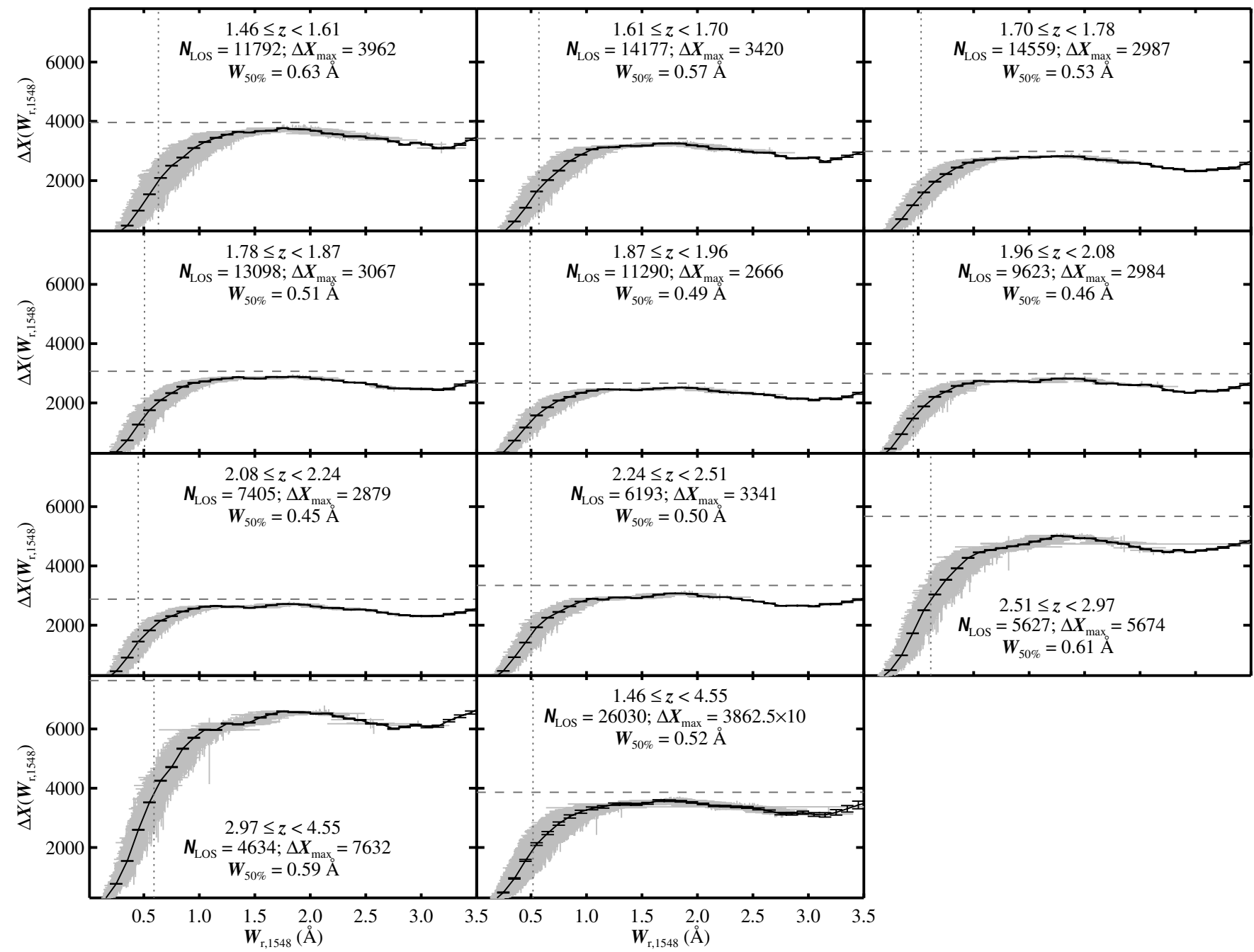

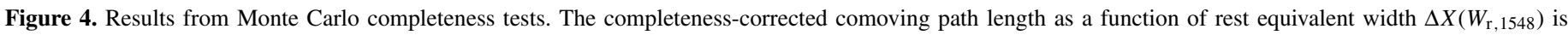

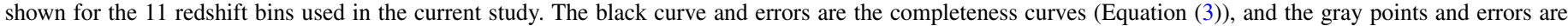

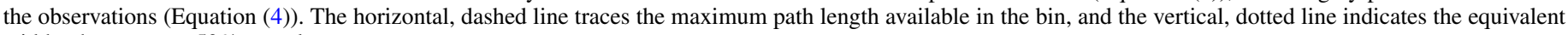
width where we are $50 \%$ complete.

they roll off at $W_{\mathrm{r}, 1548} \approx 2 \AA$. This feature results from the incompleteness in the broad (self-blended) C IV search and, to a much lesser extent $(<5 \%)$, from broad profiles being overfit by the continuum algorithm, which led them to being missed in the automated search.

The user completeness test measured our ability to correctly rate real, automatically detected doublets ("true positives") as well as the rate at which we include spurious pairs of lines as true doublets ("accepted false negatives"). We injected $\mathcal{N}_{\text {input }}=$ 5021 fake doublets with $W_{\mathrm{r}}<1 \AA$ and $\delta v_{\text {QSO }}<-5000 \mathrm{~km} \mathrm{~s}^{-1}$, and $\mathcal{N}_{\text {rec }}=3070$ were automatically recovered. Of these, we correctly rated $\mathcal{N}_{\text {accept }}=2489$. The left panels of Figure 5 show the trends of the user completeness with redshift, spectrum $\langle\mathrm{S} / \mathrm{N}\rangle$, and equivalent width.

Generally, $C_{\text {user }}$ decreases with increasing redshift and decreasing $\langle\mathrm{S} / \mathrm{N}\rangle$. Skylines become numerous at $\lambda \gtrsim 7000 \AA$, and poor sky subtraction leaves features in the spectrum that mimic absorption lines (see Figure 2). In the visual verification step, a larger fraction of real doublets are rejected due to the severe confusion. Lower $\mathrm{S} / \mathrm{N}$ spectra induces the same effect.

There were an additional 966 spurious candidates brought forth by the automated search, of which we incorrectly accepted 121. The accepted false-positive fraction peaks at $\approx 20 \%$ at $z \approx 3$ and $\langle\mathrm{S} / \mathrm{N}\rangle \approx 10$. However, our acceptance fraction grows sharply at $W_{\mathrm{r}} \lesssim 0.6 \AA$ and plateaus to $18 \%$ and $12 \%$ for $z<2.97$ and $\geqslant 2.97$, respectively. The estimated comoving line densities of accepted false positives with $W_{\mathrm{r}} \geqslant 0.6 \AA$ are $d \mathcal{N}_{\text {afp }} / d X=0.022$ and 0.020 for the low- and high-redshift bins, respectively.

We detail how we applied corrections for the accepted falsepositive rate in the next section.

\section{RESULTS}

The bulk of the analysis of the C IV sample was performed either on the whole data set or in bins in redshift space. The bins were determined empirically from the $\delta v_{\mathrm{QSO}}<-5000 \mathrm{~km} \mathrm{~s}^{-1}$ sample to have about 1500 doublets per bin, and the $\delta v_{\text {QSO }}$ cut is explained below.

\subsection{Frequency Distribution}

The equivalent width frequency distribution $f\left(W_{\mathrm{r}}\right)$ is the number of detections $\mathcal{N}_{\text {obs }}\left(W_{\mathrm{r}}\right)$ per rest equivalent width bin $\Delta W_{\mathrm{r}}$ per the total comoving path length available, in the given 

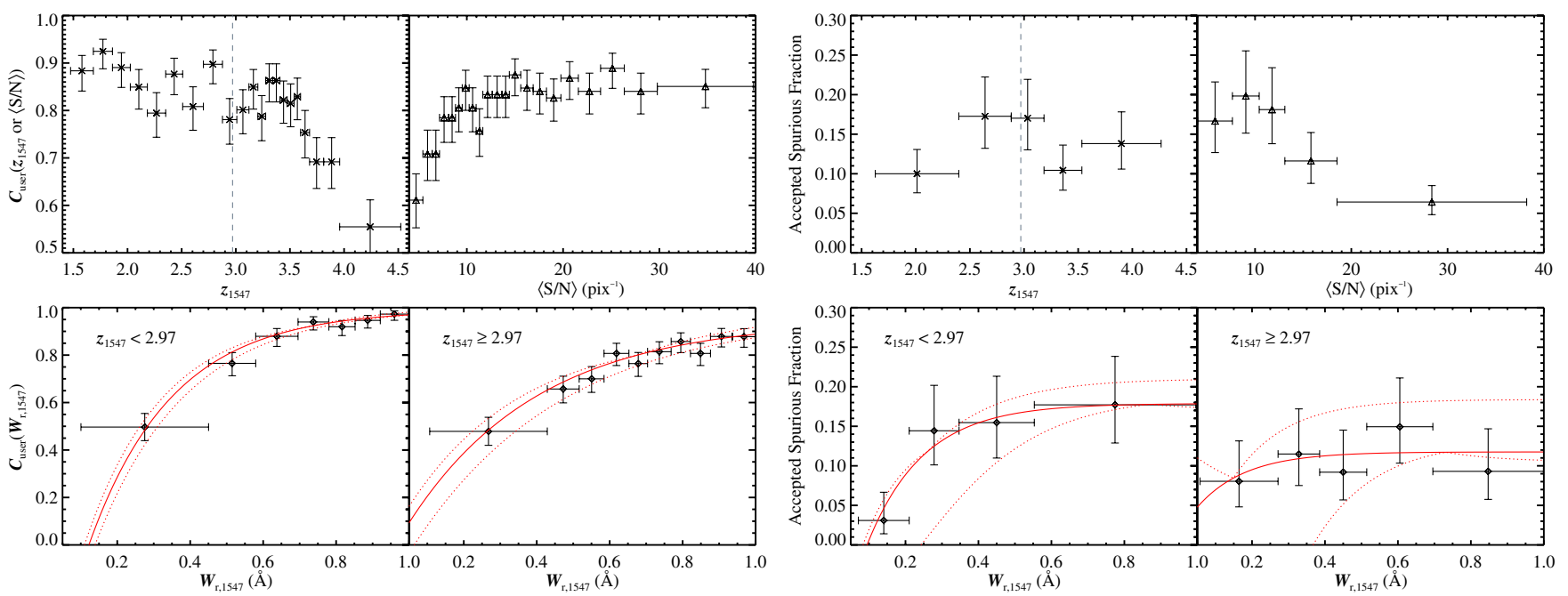

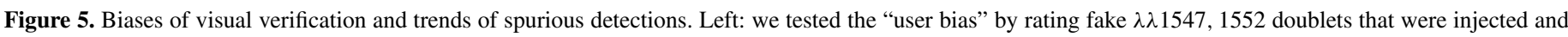

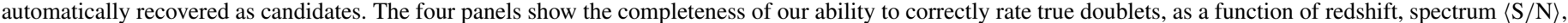

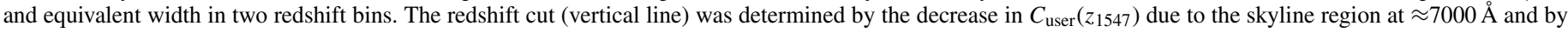

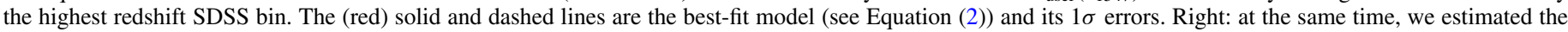

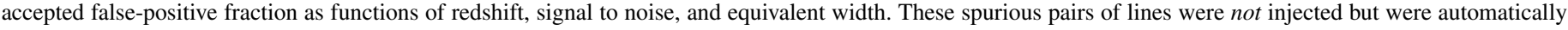
recovered. The lower panels show the best-fit model and errors to the accepted spurious fraction over $W_{\mathrm{r}, 1547}$.

(A color version of this figure is available in the online journal.)

equivalent width bin, $\Delta X\left(W_{\mathrm{r}}\right)$ :

$$
\begin{aligned}
& f\left(W_{\mathrm{r}}\right)=\frac{\mathcal{N}_{\mathrm{obs}}\left(W_{\mathrm{r}}\right)}{\Delta W_{\mathrm{r}} \Delta X\left(W_{\mathrm{r}}\right)} \\
& \sigma_{f\left(W_{\mathrm{r}}\right)}^{2}=f\left(W_{\mathrm{r}}\right)^{2}\left(\left(\frac{\sigma_{\mathcal{N}_{\mathrm{obs}}}}{\mathcal{N}_{\mathrm{obs}}\left(W_{\mathrm{r}}\right)}\right)^{2}+\left(\frac{\sigma_{\Delta X}}{\Delta X\left(W_{\mathrm{r}}\right)}\right)^{2}\right) .
\end{aligned}
$$

The error on $\mathcal{N}_{\text {obs }}$ is estimated from a Poisson distribution if $\mathcal{N}_{\text {obs }}<120$ and from a Gaussian approximation if $\mathcal{N}_{\text {obs }} \geqslant 120$. For $\Delta X\left(W_{\mathrm{r}}\right)$ and $\sigma_{\Delta X}$, we used Equation (4) with the center of the equivalent width bin being $W_{\mathrm{i}}$ and $\sigma_{W_{\mathrm{i}}}=0.5 \Delta W_{\mathrm{r}}$.

We used the maximum likelihood analysis of Cooksey et al. (2010) to fit $f\left(W_{\mathrm{r}}\right)$ with an exponential:

$$
f\left(W_{\mathrm{r}}\right)=k e^{\alpha W_{\mathrm{r}}}
$$

(see Figure 6). The normalization $k$ and scale $\alpha$ were simultaneously fit, and the errors were estimated by the maximum extent of the $1 \sigma$ error ellipse on the likelihood surface (see Figure 7). All frequency distributions were fit over the range $0.6 \AA \leqslant W_{\mathrm{r}, 1548} \leqslant \max \left[W_{\mathrm{r}, 1548}+\sigma_{W_{\mathrm{r}}}\right]$. The results were not strongly dependent on the choice of the upper limit. The exponential model is a very good description of the data, and the best-fit parameters are given in Table 4.

The best-fit parameters show smooth redshift evolution with respect to the constant $d \mathcal{N}_{\mathrm{C} \text { IV }} / d X$ curves in Figure 7 . The comoving line density is simply the integral of the frequency distribution from some limiting equivalent width $W_{\text {lim }}$ to infinity, and substituting in our exponential model, we see

$$
\left.\frac{d \mathcal{N}_{\mathrm{C} \text { IV }}}{d X}\right|_{\mathrm{fit}}=\frac{-k}{\alpha} e^{\alpha W_{\mathrm{lim}}} .
$$

By fixing $d \mathcal{N}_{\text {Civ }} / d X$, we can solve for the required normalization $k$ for any given $\alpha$. The $1 \sigma$ ellipses are elongated in the direction of the constant $d \mathcal{N}_{\mathrm{C} \text { IV }} / d X$ curves, and redshift evolution can be seen by tracking systematic change perpendicular to these curves. The lowest five redshift bins $(1.46 \leqslant z<1.96)$ fall along roughly the same constant $d \mathcal{N}_{\text {C IV }} / d X$ curve. The next three highest redshift bins $(1.96 \leqslant z<2.51)$ have slightly smaller line densities. Then there is almost a factor of two drop over the highest two redshift bins $(2.51 \leqslant z<4.55)$.

Since the accepted false-positive rate is essentially constant at $W_{\mathrm{r}} \geqslant 0.6 \AA$ (see Section 3.4), the frequency distribution of accepted false positives is a scaled-down version of the measured frequency distribution. Therefore, we scale the original $f_{0}\left(W_{\mathrm{r}}\right)$ as follows:

$$
f\left(W_{\mathrm{r}}\right)=\left(1-\frac{d \mathcal{N}_{\mathrm{afp}} / d X}{d \mathcal{N}_{\mathrm{C} \text { IV }} / d X}\right) f_{0}\left(W_{\mathrm{r}}\right)
$$

and propagate the errors. This results in a decrease of $\approx 6 \%-12 \%$, depending on the redshift. For the exponential fits, we scale the best-fit normalization $k_{0}$ in a similar fashion:

$$
k=\left(1-\frac{d \mathcal{N}_{\mathrm{afp}} / d X}{\left(d \mathcal{N}_{\mathrm{C} \text { IV }} / d X\right)_{\mathrm{fit}}}\right) k_{0},
$$

but the denominator is the integrated line density from the exponential model (Equation (7)). We report the propagated errors in Table 4 and in the text. However, since we cannot compute the change in the likelihood surface, we only shift the ellipses in Figures 7 and 8.

\subsubsection{Effect of Blending and "Intrinsic" Absorbers}

As mentioned in Section 2.5, blended profiles are an issue for this survey, which relies on automated procedures and boxcar summation to measure equivalent widths. We ran 1000 Monte Carlo simulations to estimate the net effect of blending. There were over $10^{6}(17 \%)$ simulated doublets recovered in the completeness tests that had measured equivalent widths more than $3 \sigma$ larger than the input value, indicative of blending. We measured the median $\left(\left\langle W_{\text {blend }}\right\rangle=0.2 \AA\right)$ and standard deviation $\left(\sigma_{W_{\text {blend }}}=0.5 \AA\right)$ of the distribution, where $W_{\text {blend }}=$ $W_{\mathrm{r}, \text { rec }}-\left(W_{\mathrm{r} \text {,input }}+3 \sigma_{W_{\mathrm{r}, \text { rec }}}\right)$. For each realization, we tested 


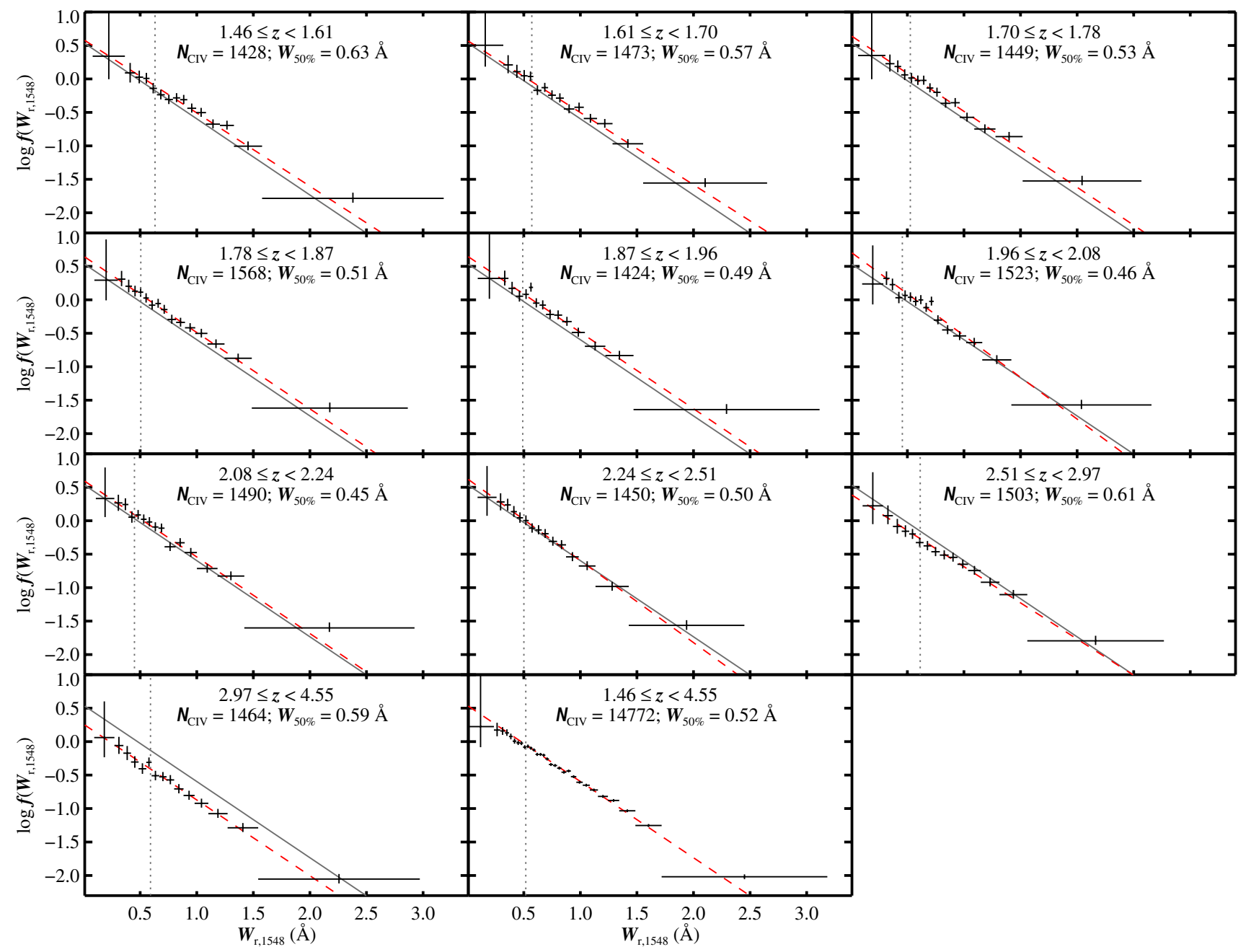

Figure 6. Equivalent width frequency distributions. The maximum likelihood fits of an exponential function are the dashed (red) lines, for each redshift bin, and the solid (gray) line, for the full sample. There is little evolution with redshift. The observations have been completeness corrected, and the redshift-specific 50\% completeness limits are the vertical dotted lines.

(A color version of this figure is available in the online journal.)

Table 4

C Iv Results Summary

\begin{tabular}{|c|c|c|c|c|c|c|c|c|c|c|c|}
\hline $\begin{array}{l}(1) \\
\langle z\rangle\end{array}$ & $\begin{array}{l}(2) \\
z_{\lim }\end{array}$ & $\begin{array}{c}(3) \\
\mathcal{N}_{\text {obs }}\end{array}$ & $\begin{array}{c}(4) \\
\Delta X_{\max }\end{array}$ & $\begin{array}{c}(5) \\
W_{50 \%} \\
(\AA)\end{array}$ & $\begin{array}{c}(6) \\
d \mathcal{N}_{\text {CIV }}^{(6)} / d z\end{array}$ & $\begin{array}{c}(7) \\
d \mathcal{N}_{\text {CIV }} / d X\end{array}$ & $\begin{array}{c}(8) \\
\Omega_{\mathrm{CIV}} \\
\left(\times 10^{-8}\right)\end{array}$ & $\begin{array}{l}(9) \\
\mathcal{N}_{\text {fit }}\end{array}$ & $\begin{array}{c}(10) \\
k \\
\left(\AA^{-1}\right)\end{array}$ & $\begin{array}{c}(11) \\
\alpha \\
\left(\AA^{-1}\right)\end{array}$ & $\begin{array}{l}(12) \\
\chi_{\text {red }}^{2}\end{array}$ \\
\hline 1.96274 & {$[1.46623,4.54334]$} & 14772 & 38624 & 0.52 & $0.92_{-0.01}^{+0.02}$ & $0.275_{-0.004}^{+0.004}$ & $1.71_{-0.20}^{+0.20}$ & 8918 & $3.49_{-0.30}^{+0.31}$ & $-2.62_{-0.04}^{+0.04}$ & 0.577 \\
\hline 1.55687 & {$[1.46623,1.60986]$} & 1428 & 3962 & 0.63 & $0.95_{-0.03}^{+0.03}$ & $0.332_{-0.010}^{+0.011}$ & $2.18_{-0.39}^{+0.39}$ & 1025 & $3.87_{-0.82}^{+0.94}$ & $-2.52_{-0.12}^{+0.11}$ & 1.560 \\
\hline 1.65971 & {$[1.61004,1.69993]$} & 1473 & 3420 & 0.57 & $1.00_{-0.03}^{+0.04}$ & $0.336_{-0.011}^{+0.012}$ & $2.10_{-0.32}^{+0.32}$ & 956 & $3.77_{-0.82}^{+0.95}$ & $-2.48_{-0.13}^{+0.12}$ & 0.876 \\
\hline 1.73999 & {$[1.70035,1.78000]$} & 1449 & 2987 & 0.53 & $1.08_{-0.04}^{+0.04}$ & $0.356_{-0.012}^{+0.013}$ & $2.17_{-0.28}^{+0.28}$ & 902 & $4.46_{-1.06}^{+1.24}$ & $-2.61_{-0.14}^{+0.14}$ & 1.672 \\
\hline 1.82360 & {$[1.78007,1.86985]$} & 1568 & 3067 & 0.51 & $1.09_{-0.04}^{+0.04}$ & $0.351_{-0.011}^{+0.012}$ & $2.11_{-0.24}^{+0.23}$ & 932 & $4.49_{-1.04}^{+1.20}$ & $-2.63_{-0.13}^{+0.13}$ & 1.291 \\
\hline 1.91460 & {$[1.87000,1.95998]$} & 1424 & 2665 & 0.49 & $1.12_{-0.04}^{+0.04}$ & $0.355_{-0.012}^{+0.013}$ & $2.15_{-0.23}^{+0.23}$ & 824 & $4.53_{-1.09}^{+1.28}$ & $-2.63_{-0.14}^{+0.14}$ & 1.832 \\
\hline 2.01778 & {$[1.96002,2.07997]$} & 1523 & 2983 & 0.46 & $1.04_{-0.04}^{+0.04}$ & $0.322_{-0.011}^{+0.012}$ & $1.86_{-0.17}^{+0.17}$ & 852 & $5.18_{-1.51}^{+1.76}$ & $-2.87_{-0.15}^{+0.15}$ & 2.838 \\
\hline 2.15320 & {$[2.08009,2.23997]$} & 1490 & 2878 & 0.45 & $1.04_{-0.04}^{+0.04}$ & $0.312_{-0.011}^{+0.012}$ & $1.92_{-0.17}^{+0.17}$ & 807 & $4.01_{-1.04}^{+1.21}$ & $-2.63_{-0.14}^{+0.14}$ & 3.267 \\
\hline 2.35608 & {$[2.24015,2.50914]$} & 1450 & 3341 & 0.50 & $0.96_{-0.03}^{+0.04}$ & $0.276_{-0.010}^{+0.011}$ & $1.61_{-0.18}^{+0.18}$ & 775 & $4.26_{-1.37}^{+1.62}$ & $-2.82_{-0.16}^{+0.15}$ & 1.296 \\
\hline 2.72298 & {$[2.51028,2.96976]$} & 1503 & 5673 & 0.61 & $0.83_{-0.03}^{+0.03}$ & $0.221_{-0.008}^{+0.009}$ & $1.44_{-0.22}^{+0.22}$ & 969 & $2.48_{-0.65}^{+0.74}$ & $-2.49_{-0.12}^{+0.12}$ & 0.837 \\
\hline 3.25860 & {$[2.97005,4.54334]$} & 1464 & 7632 & 0.59 & $0.59_{-0.02}^{+0.02}$ & $0.145_{-0.005}^{+0.006}$ & $0.87_{-0.13}^{+0.13}$ & 876 & $1.82_{-0.65}^{+0.75}$ & $-2.61_{-0.13}^{+0.13}$ & 1.548 \\
\hline
\end{tabular}

Notes. Summary of the most common redshift bins and data used for the various analyses. Columns 1 and 2 give the median, minimum, and maximum redshifts for the observed number of doublets (Column 3), and the maximum comoving path length in the redshift bin is given in Column 4 . The 50\% completeness limit from the Monte Carlo tests is in Column 5. The redshift and comoving absorber line densities for $W_{\mathrm{r}} \geqslant 0.6 \AA$ are in Columns 6 and 7 . In Column 8 , the $\Omega_{\mathrm{C} \text { Iv }}$ from summing the mass in the $W_{\mathrm{r}} \geqslant 0.6 \AA$ absorbers is a lower limit, since the majority of absorbers are saturated. The frequency distribution was fit with an exponential $f\left(W_{\mathrm{r}}\right)=k \exp \left(\alpha W_{\mathrm{r}}\right)$ for $\mathcal{N}_{\text {fit }}$ absorbers with $W_{\mathrm{r}} \geqslant 0.6 \AA$ (Column 9), and the best-fit parameters are given in Columns 10 and 11 . The reduced $\chi^{2}$ from the best fit and $f\left(W_{\mathrm{r}}\right.$ ) (in bins with $\approx 100$ doublets each) is given in Column 12 . 


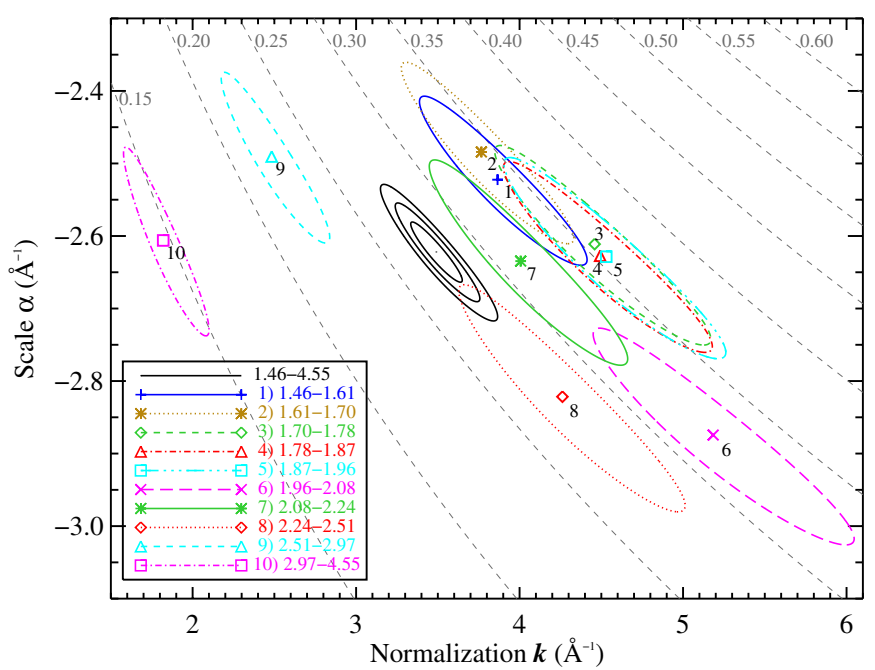

Figure 7. Best-fit $f\left(W_{\mathrm{r}}\right)$ parameters and errors. We fit the frequency distribution with an exponential function (see Equation (6)). The best-fit normalization $k$ and scale $\alpha$ and the $1 \sigma$ error ellipses are plotted for the 10 small redshift bins (numbered points); the $1 \sigma, 2 \sigma$, and $3 \sigma$ contours are shown for the fit to the full sample (black ellipses). The best-fit parameters smoothly evolve with redshift, as seen by comparing the ellipses with the constant $d \mathcal{N}_{\mathrm{C} \text { Iv }} / d X$ curves (gray, dashed lines).

(A color version of this figure is available in the online journal.)

the worst-case scenario by decreasing the equivalent width of a random $20 \%$ of the absorbers, which was the largest blended fraction estimated in Section 2.5. The magnitude of the decrease was drawn randomly from the half of a Gaussian distribution below its mean, set to $\left\langle W_{\text {blend }}\right\rangle$, with standard deviation $\sigma_{W_{\text {blend }}}$. Then, the new sample was fit with an exponential $f\left(W_{\mathrm{r}}\right)$ model. The median best-fit parameters from these Monte Carlo simulations were in very good agreement with those in Table 4, and the standard deviation of the Monte Carlo results was 10 times smaller than the quoted uncertainties. These simulations assessed the worst-case scenario, by taking the largest estimated fraction of blended lines (see Section 2.5) and only decreasing the equivalent widths (as opposed to also re-sampling the other $80 \%$ of the doublets).

In addition, the completeness corrections account for blending since we compiled the curves in Figure 4 with respect to the measured - as opposed to input-equivalent widths. There were other absorption lines in the spectra, and the randomly placed simulated profiles were blended at a realistic rate, as seen by the agreement of the blended fraction from our visual estimate (Section 2.5) and from the $10^{6}$ simulated doublets described above. Thus, our results are robust to the effects of blending.

We tested the effect of the $\delta v_{\text {QSO }}$ cut on our results by increasing $\delta v_{\text {QSO }}$ and re-fitting the full redshift sample (see Figure 8). Systems close to the background QSO could potentially be high velocity, intrinsic absorbers and/or affected by quasar clustering, enrichment, and/or local ionization. The general trend for $\delta v_{\mathrm{QSO}}<-4000 \mathrm{~km} \mathrm{~s}^{-1}$ is decreasing normalization (i.e., $k$ ) with nearly constant shape $(\alpha)$. The decrease in $k$ is due to the number of absorbers decreasing faster than the path length up to at least $\delta v_{\mathrm{QSO}}=-15,000 \mathrm{~km} \mathrm{~s}^{-1}$. For example, with $\delta v_{\mathrm{QSO}}=-10,000 \mathrm{~km} \mathrm{~s}^{-1}$, we have $17.5 \%$ less path length but $25 \%$ fewer $W_{\mathrm{r}} \geqslant 0.6 \AA$ doublets. This trend is partially explained by quasars residing in dense environments (e.g., Prochaska et al. 2011), where the chance of intersecting a metal-enriched galaxy halo is increased.

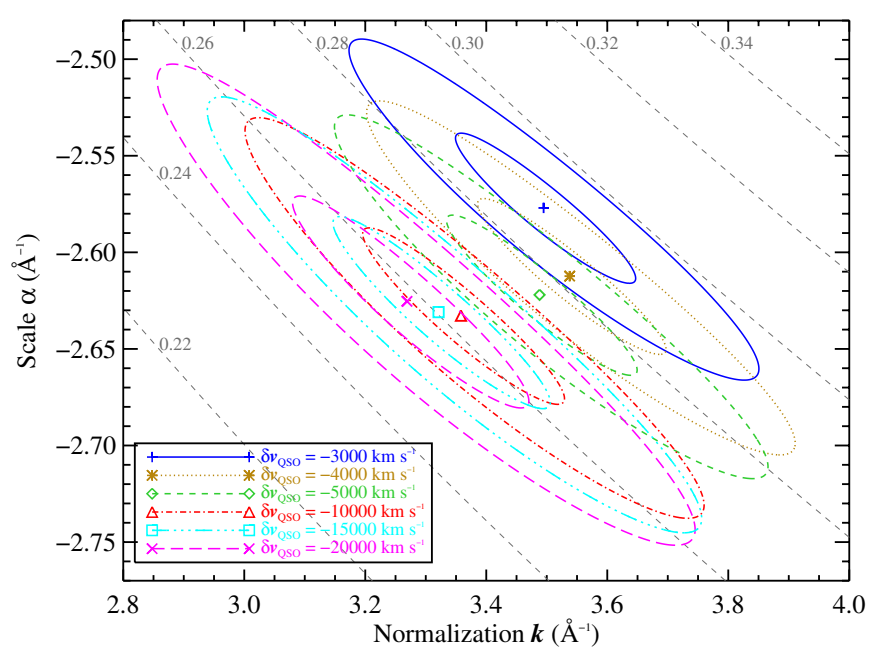

Figure 8. Best-fit $f\left(W_{\mathrm{r}}\right)$ parameters for different $\delta v_{\mathrm{QSO}}$ values. We tested the effects of the $\delta v_{\mathrm{QSO}}$ cut on the fit to the full C IV sample. The contours are $1 \sigma$ and $3 \sigma$, and the gray, dashed lines are constant $d \mathcal{N}_{\mathrm{C} \text { IV }} / d X$ curves. We adopt $\delta v_{\mathrm{QSO}}=-5000 \mathrm{~km} \mathrm{~s}^{-1}$ for the main analyses.

(A color version of this figure is available in the online journal.)

However, this effect would not dominate out to $\delta v_{\mathrm{QSO}}=$ $-10,000 \mathrm{~km} \mathrm{~s}^{-1}$. The lack of "convergence" at very large $\delta v_{\mathrm{QSO}}$ may be partially due to poorly measured quasar redshifts. Hewett $\&$ Wild (2010) re-measured the majority of the redshifts for the Schneider et al. (2010) DR7 QSOs, with better automated routines. Adopting the Hewett \& Wild (2010) redshifts affects over $80 \%$ of the total $16,459 \mathrm{C}$ IV systems. The majority of the quasar redshifts increased, with a median $\left\langle\delta v_{\mathrm{em}}\right\rangle=$ $381 \mathrm{~km} \mathrm{~s}^{-1}$, standard deviation $764 \mathrm{~km} \mathrm{~s}^{-1}$, and maximal extent $-4200 \mathrm{~km} \mathrm{~s}^{-1} \lesssim \delta v_{\mathrm{em}} \lesssim+5700 \mathrm{~km} \mathrm{~s}^{-1}$. In addition, there are redshift-dependent fluctuations in $\delta v_{\mathrm{em}}$. Using the Hewett \& Wild (2010) redshifts would affect our analysis; however, the change in the total sample size, given a $\delta v_{\mathrm{QSO}}$ cut, is less than $2 \%$. Given this small fraction and the incompleteness of the new redshifts, we chose to continue with the Schneider et al. (2010) redshifts, but we adopt $\delta v_{\mathrm{QSO}}=-5000 \mathrm{~km} \mathrm{~s}^{-1}$ for the bulk of our analyses which reduces the sample to 14,772 doublets.

\subsection{IV Absorber Line Density}

We directly measured the absorber line density for doublets with $W_{\mathrm{r}} \geqslant W_{\text {lim }}$ as follows:

$$
\begin{aligned}
\frac{d \mathcal{N}_{\mathrm{CIV}}}{d X}\left(W_{\mathrm{r}} \geqslant W_{\lim }\right) & =\frac{\mathcal{N}_{\mathrm{C}}\left(W_{\mathrm{r}}\right)}{\Delta X(z)} \\
\sigma_{d \mathcal{N} / d X}^{2} & =\left(\frac{\sigma_{\mathcal{N}_{\mathrm{C}}}}{\Delta X(z)}\right)^{2} .
\end{aligned}
$$

The completeness-corrected number of absorbers in any given bin is the completeness-weighted sum of the observed absorbers in the bin:

$$
\begin{aligned}
\mathcal{N}_{\mathrm{C}}\left(W_{\mathrm{r}}\right) & =\sum_{W_{\mathrm{i}} \geqslant W_{\mathrm{r}}-0.5 \Delta W_{\mathrm{r}}}^{W_{\mathrm{i}}<W_{\mathrm{r}}+0.5 \Delta W_{\mathrm{r}}} \frac{1}{C\left(W_{\mathrm{i}}\right)} \\
\sigma_{\mathcal{N}_{\mathrm{C}}}^{2} & =\sigma_{\mathcal{N}_{\text {obs }}}^{2}+\sum_{W_{\mathrm{i}} \geqslant W_{\mathrm{r}}-0.5 \Delta W_{\mathrm{r}}}^{W_{\mathrm{i}}<W_{\mathrm{r}}+0.5 \Delta W_{\mathrm{r}}}\left(\frac{\sigma_{C\left(W_{\mathrm{i}}\right)}}{C\left(W_{\mathrm{i}}\right)^{2}}\right)^{2} .
\end{aligned}
$$

Again, $\mathcal{N}_{\text {obs }}$ is the contribution of the actual observed number of absorbers in the given $W_{\mathrm{r}}$ bin, and the completeness-corrected 


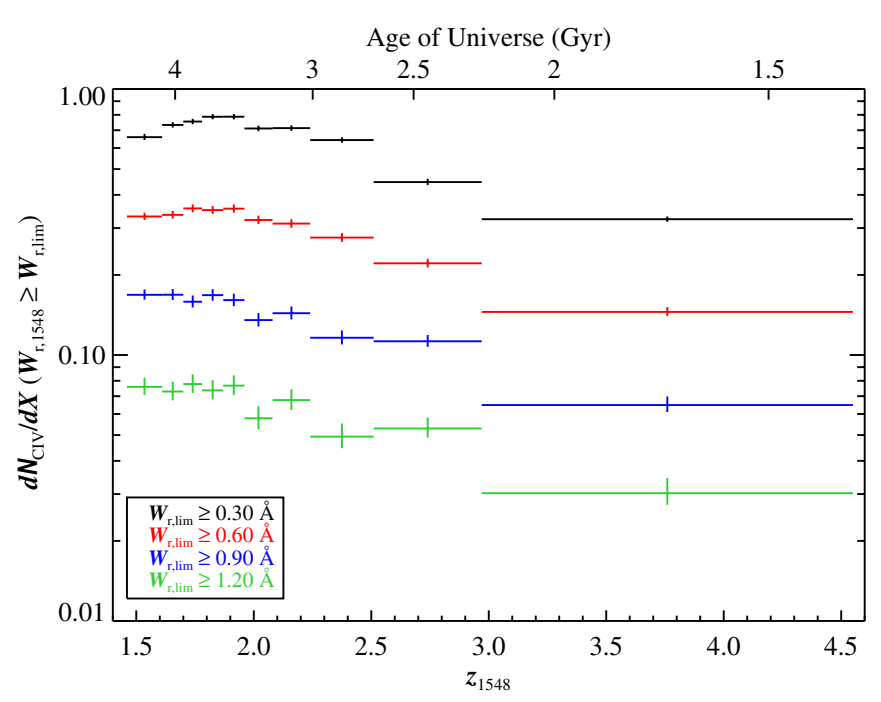

Figure 9. Comoving C IV line density evolution. The number of absorbers per comoving path length increases steadily and consistently from $z_{1548}=4.5 \rightarrow$ $\approx 1.74$. As expected from the nearly unchanging nature in the shape of $f\left(W_{\mathrm{r}}\right)$ (see Figure 6), there is little dependence on $W_{\mathrm{r}, \mathrm{lim}}$, noting that we are typically $50 \%$ at $W_{\mathrm{r}} \approx 0.6 \AA$.

(A color version of this figure is available in the online journal.)

number $\mathcal{N}_{\mathrm{C}} \geqslant \mathcal{N}_{\text {obs }}$. The error $\sigma_{\mathcal{N}_{\text {obs }}}$ is estimated from a Poisson distribution if $\mathcal{N}_{\text {obs }}<120$ and from a Gaussian approximation if $\mathcal{N}_{\text {obs }} \geqslant 120$.

We subtract $d \mathcal{N}_{\text {afp }} / d X$ from all quoted $d \mathcal{N}_{\text {C Iv }} / d X$ values, in the appropriate redshift bins (see Section 3.4) and add the errors in quadrature. For $W_{\mathrm{r}} \geqslant 0.6 \AA, d \mathcal{N}_{\mathrm{afp}} / d X=0.020_{-0.002}^{+0.003}$ $(1.4<z<4.6) ; 0.022_{-0.004}^{+0.005}(z<2.97)$; and $0.020_{-0.003}^{+0.004}$ $(z \geqslant 2.97)$.

We present $d \mathcal{N}_{\mathrm{C} \text { IV }} / d X$ for different equivalent width limits in Figure 9. The line density shows little differential evolution based on $W_{\mathrm{r}, \mathrm{lim}}$, as expected from the consistent shape of the frequency distributions over time (Figure 6). For $W_{\mathrm{r}} \geqslant 0.6 \AA$, the inverse-variance weighted average $d \mathcal{N}_{\mathrm{C}}$ IV $/ d X=0.350 \pm$ 0.005 for $1.46 \leqslant z<1.96$ (or the lowest five redshift bins). This average is $2.37 \pm 0.09$ times larger than the highest redshift $d \mathcal{N}_{\text {C IV }} / d X$ at $z=3.76$. Though the magnitude of the increase is modest, the detection is a $>20 \sigma$ result. Thus, the line density grows consistently and smoothly from $z_{1548}=4.5 \rightarrow \approx 1.74$, then plateaus at $d \mathcal{N}_{\text {C IV }} / d X \approx 0.34$ until $z=1.46$, as expected from the best-fit $f\left(W_{\mathrm{r}}\right)$ parameters (see Figure 7).

There is a known bias in the SDSS quasar color selection that leads to an excess of Lyman-limit systems at $3 \lesssim z_{\mathrm{QSO}} \lesssim 3.5$ (Prochaska et al. 2010; Worseck \& Prochaska 2011), which likely increases the incidence of strong metal-line absorption systems at these redshifts. Therefore, there is potential for the decrease in the highest SDSS bin to be even larger, but the effect of the color selection on the C IV sample is beyond the scope of this paper.

\subsection{Comparison with Previous Results}

To fairly compare to other surveys, we applied various $W_{\mathrm{r}}$ cuts to our complete sample, chosen to match the corresponding cuts of prior surveys.

To compare $f\left(W_{\mathrm{r}}\right)$, we converted published column density frequency distributions fits from Songaila (2001) and D'Odorico et al. (2010) to equivalent width frequency distributions by assuming the linear curve of growth and mapping $f\left(N_{\mathrm{C}_{\text {IV }}}\right)$ directly to $f\left(W_{\mathrm{r}, 1548}\right): f\left(W_{\mathrm{r}}\right)=f\left(N_{\mathrm{C} \text { IV }}\right) d N_{\mathrm{C} \text { IV }} / d W_{\mathrm{r}}$. We adjusted for cosmology as necessary. A single-component cloud becomes saturated and nonlinear at $\log N_{\mathrm{CIV}} \approx 14$, which translates to $W_{\mathrm{r}, 1548} \approx 0.6 \AA$, where we typically are $\approx 50 \%$ complete.

While we have good statistics on the rare, strong absorbers, we suffer from incompleteness at $W_{\mathrm{r}} \lesssim 0.6 \AA$. Songaila (2001) and D'Odorico et al. (2010) were smaller, higher-resolution, higher-S/N studies, and they were complete to very low $W_{\mathrm{r}}$ but suffered from sample variance at larger equivalent widths. However, Figure 10 shows that our results are consistent with each other in the overlap region. Our best-fit parameters to the exponential $f\left(W_{\mathrm{r}}\right)$ in the D'Odorico et al. (2010) and Songaila (2001) redshift bins were, respectively: $\alpha=-2.65_{-0.04}^{+0.04} \AA^{-1}$ and $k=3.72_{-0.35}^{+0.37} \AA^{-1}$ for $1.6 \leqslant z \leqslant 3.6$ with 7884 systems; and $\alpha=-2.58_{-0.13}^{+0.13} \AA^{-1}$ and $k=2.29_{-0.69}^{+0.80} \AA^{-1}$ for $2.9 \leqslant z \leqslant 3.54$ with 878 systems.

We compared the SDSS redshift and comoving line densities with the literature (Steidel 1990; Barlow \& Tytler 1998;
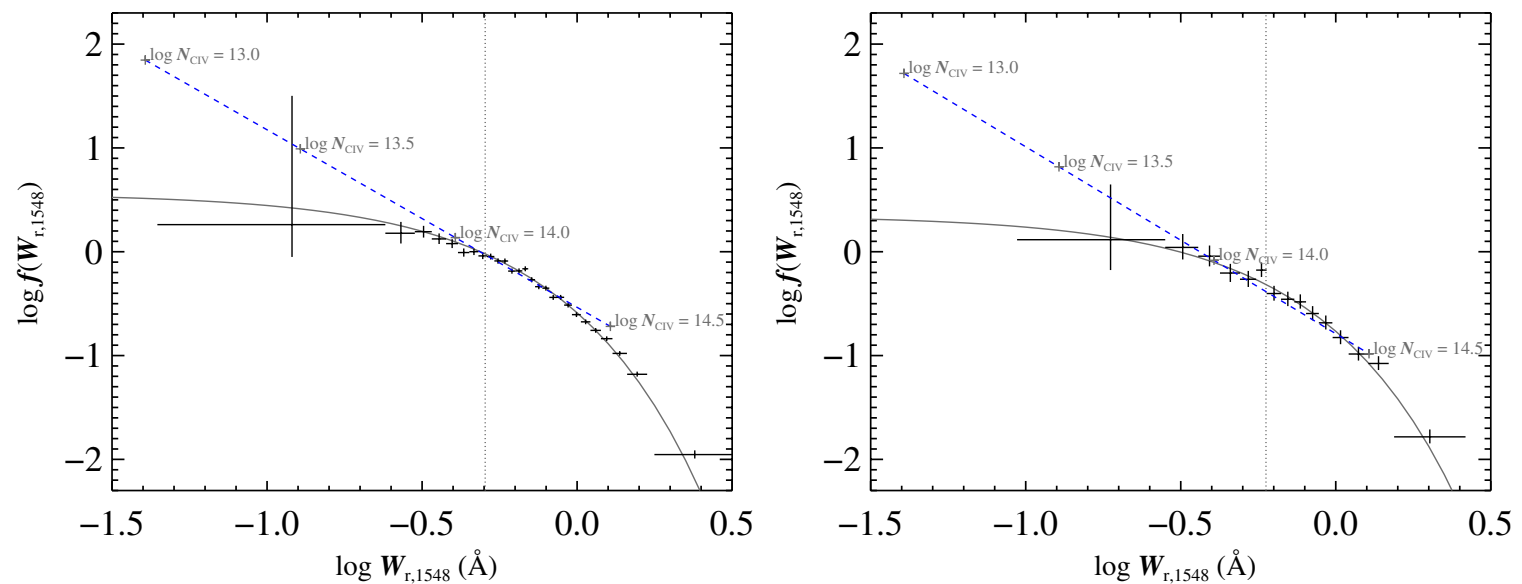

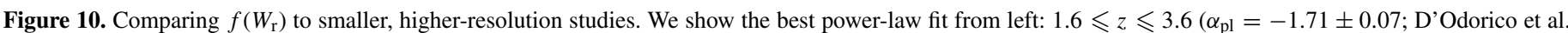

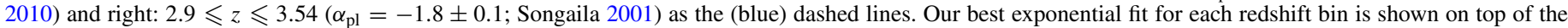

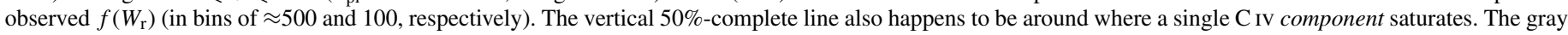
plus signs indicate the equivalent widths where the labeled column densities fall.

(A color version of this figure is available in the online journal.) 

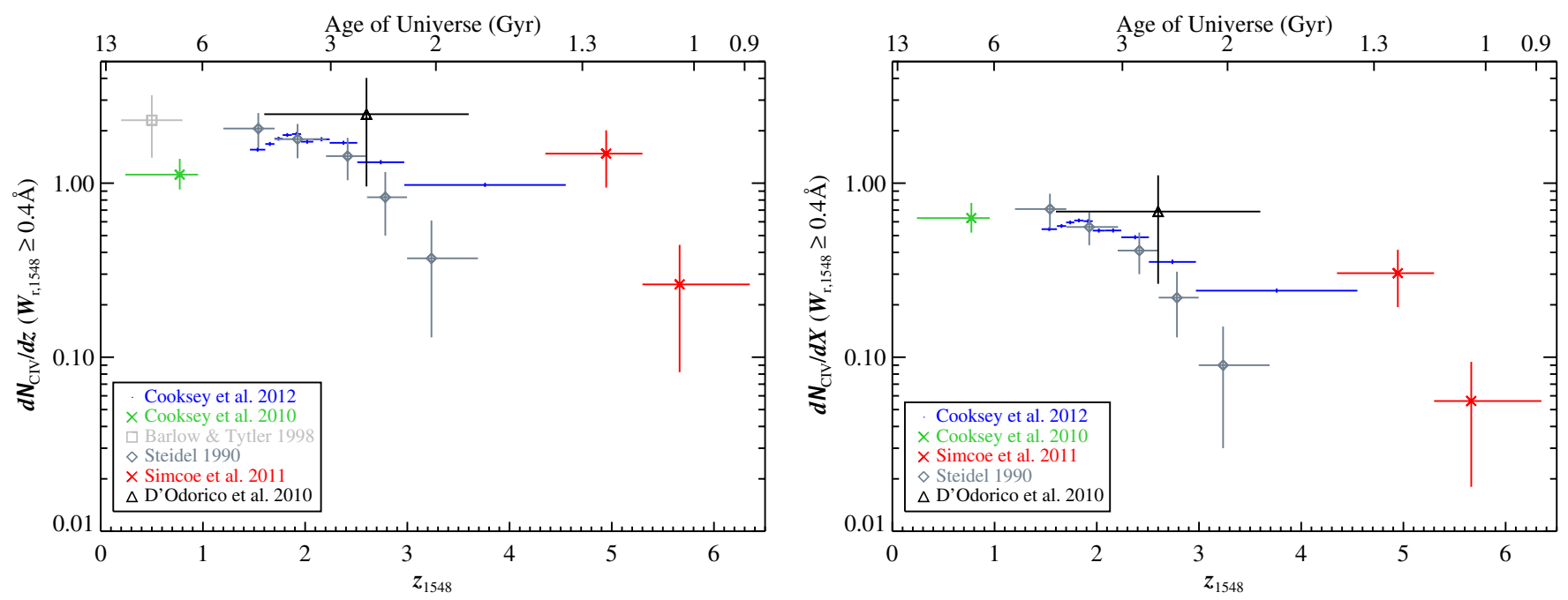

Figure 11. Redshift (left) and comoving (right) $\mathrm{C}_{\mathrm{IV}}$ absorber line densities. We compare apples-to-apples $d \mathcal{N}_{\mathrm{C} \text { IV }} / d z$ with $W_{\mathrm{r}} \geqslant 0.4 \AA$ with five surveys: Barlow \& Tytler (1998, light gray square), Cooksey et al. (2010, green cross), D’Odorico et al. (2010, black triangle), Steidel (1990, gray diamonds), and Simcoe et al. (2011, red crosses). The comoving line density steadily increases from high to low redshift. The growth in $d \mathcal{N}_{\mathrm{C} \text { Iv }} / d X$ indicates a consistent increase with time in the product of the comoving number density and the physical cross-section of the absorbing clouds.

(A color version of this figure is available in the online journal.)

Cooksey et al. 2010; D’Odorico et al. 2010; Simcoe et al. 2011). For D'Odorico et al. (2010), we used their best-fit $f\left(N_{\mathrm{C}_{\text {IV }}}\right)$ values to calculate $d \mathcal{N}_{\text {C IV }} / d X$ and $d \mathcal{N}_{\text {CIV }} / d z=$ $\left(d \mathcal{N}_{\text {C Iv }} / d X\right)(d X / d z)$ for $\log N_{\text {CIV }} \geqslant 14$. For Barlow \& Tytler (1998) and Steidel (1990), we estimated $d \mathcal{N}_{\text {Civ }} / d X=$ $\left(d \mathcal{N}_{\text {C IV }} / d z\right)(d X / d z)^{-1}$. The Steidel (1990) results match ours well for the range where the author was fairly complete but with $\approx 20 \%$ uncertainty, compared to our $\approx 2 \%$ errors (see Figure 11 ). Extending the redshift coverage by including the low-redshift measurements of Barlow \& Tytler (1998) and Cooksey et al. (2010) and the high-redshift values from Simcoe et al. (2011), we see that $d \mathcal{N}_{\text {C Iv }} / d X$ has steadily increased by, roughly, a factor of 10 from $z_{1548}=6 \rightarrow 0$.

\section{DISCUSSION}

\subsection{IV Evolution}

We show the best measurements of $d \mathcal{N}_{\text {C }} / d X$ for $W_{\mathrm{r}} \geqslant$ $0.6 \AA$ and $0<z<6.5$ in the top panel of Figure 12. ${ }^{10}$ The comoving line density relates to the comoving volume density of absorbing clouds $n_{\text {com }}$ and their physical cross-section $\sigma_{\text {phys }}$ :

$$
\frac{d \mathcal{N}_{\mathrm{C} \text { IV }}}{d X}=\frac{c}{H_{0}} n_{\text {com }} \sigma_{\text {phys }} .
$$

Thus, the roughly order-of-magnitude increase in $d \mathcal{N}_{\text {C Iv }} / d X$ from high-to-low redshift means the product $n_{\text {com }} \sigma_{\text {phys }}$ has increased by a factor of $\approx 10$. We know the metallicity of the universe has steadily increased over cosmic time, so the possible number of C IV-absorbing clouds (i.e., $n_{\text {com }}$ ) has likely increased. At least some $\mathrm{C}$ IV absorption traces galaxy halos at low (Chen et al. 2001) and high redshift (Adelberger et al. 2005; Martin et al. 2010; Steidel et al. 2010). Since galaxies (and their halos) have likely grown over cosmic time, increases in both $\sigma_{\text {phys }}$ and $n_{\text {com }}$ appear to contribute to the increase in $d \mathcal{N}_{\mathrm{C} \text { IV }} / d X$.

\footnotetext{
${ }^{10}$ For $z<1$, we summed the eight $W_{\mathrm{r}} \geqslant 0.6 \AA$ systems in the $H S T$ sample (Cooksey et al. 2010) to get $d \mathcal{N}_{\mathrm{C} \text { IV }} / d X=0.63_{-0.11}^{+0.14}$. For $z>4$, we have $d \mathcal{N}_{\text {C IV }} / d X=0.212 \pm 0.092$ for $z=4.94$ (two systems) and $0.026 \pm 0.027$ for $z=5.66$ (1) from the FIRE sample (Simcoe et al. 2011).
}

Adelberger et al. (2005) reported that almost all $\log N_{\mathrm{CIV}} \geqslant$ 14 absorbers arise within $\approx 80 \mathrm{kpc}$ of Lyman-break galaxies (LBGs) at $2 \lesssim z \lesssim 3$. The evidence included individual strong absorber-LBG pairs; strong absorption in stacked spectra of close background galaxies, shifted to the rest frame of the foreground galaxies; and similar LBG-C IV cross-correlation and LBG autocorrelation functions, suggesting they have the same spatial distribution.

Steidel et al. (2010) increased the LBG-LBG pairs for stacking analysis, and they measured average $W_{\mathrm{r}, 1548}=0.13 \pm 0.05 \AA$ and $1.18 \pm 0.15 \AA$ at distances $b=63 \mathrm{kpc}$ and $103 \mathrm{kpc}$, respectively. Interpolating between these two measurements, an average $W_{\mathrm{r}, 1548}=0.6 \AA$ system would reside at $b \approx$ $85 \mathrm{kpc}$. The Steidel et al. (2010) LBG sample went as faint as $\approx 0.3 L^{*}$, assuming the luminosity function of Reddy \& Steidel (2009), and since they used stacks of galaxy spectra, their analysis included the effects of partial covering fractions.

Since the $W_{\mathrm{r}} \geqslant 0.6 \AA \mathrm{C}_{\text {IV }}$ absorbers in our sample have $\log N_{\text {CIV }} \gtrsim 14$, we used the comoving number density of UV-selected galaxies, to estimate the typical galaxy-C IV crosssection over time with Equation (12). We measured $n_{\text {com.uv }}$ with the UV luminosity functions from Oesch et al. (2010), Reddy \& Steidel (2009), and Bouwens et al. (2007), which covered several smaller redshift bins spanning $0.5<z<2,1.9<z<3.4$, and $3.8 \lesssim z \lesssim 5.9$, respectively. For each luminosity function, we integrated the best-fit Schechter function down to $0.5 \mathrm{~L}^{*}$ or $\approx 0.75$ mag fainter than the published $M_{\mathrm{UV}}^{*}$, which ranged between $\approx-19$ and $-21 \mathrm{mag}$. We estimated the $n_{\text {com, Uv }}$ errors with Monte Carlo simulations.

The UV-selected galaxy number density, $n_{\mathrm{com}, \mathrm{UV}}$, may increase by a factor of two to three from $z \approx 6 \rightarrow 0$, but the uncertainties are large (see Figure 12, middle panel). Applying $n_{\text {com,UV }}$ and $d \mathcal{N}_{\text {CIV }} / d X$ to Equation (12), we estimated $\sigma_{\text {phys }}$, the physical galaxy-C IV cross-section (Figure 12, lower panel). For some redshift bins, multiple luminosity functions could be used in conjunction with our $d \mathcal{N}_{\text {C }} / d X$, and we show all resulting values but highlight the preferred values with filled symbols. 

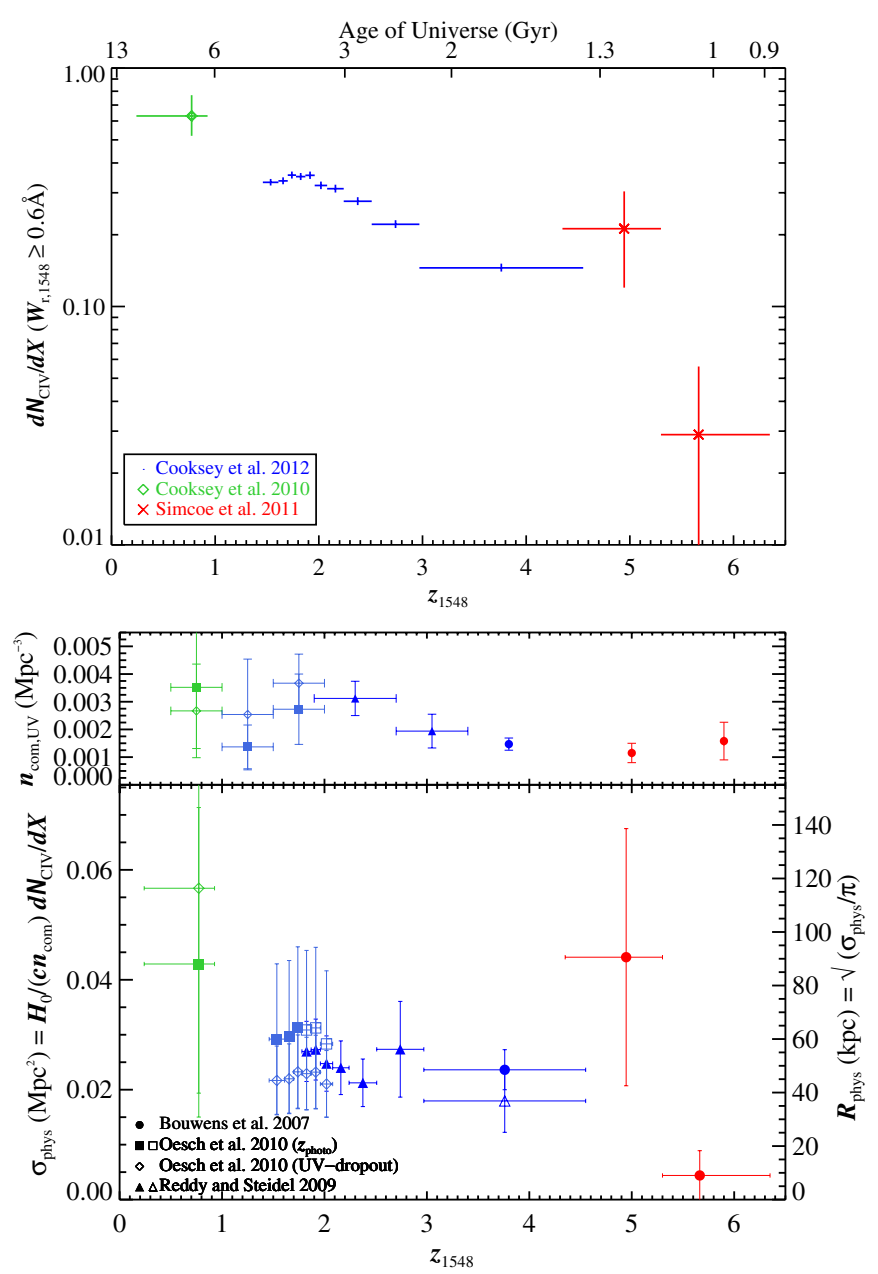

Figure 12. Evolving nature of C IV absorbers. Top: by combining the current SDSS values with those from Cooksey et al. (2010) and Simcoe et al. (2011), we show that $d \mathcal{N}_{\mathrm{C} \text { IV }} / d X$ for $W_{\mathrm{r}, 1548} \geqslant 0.6 \AA$ has increased 10-fold, roughly, from $z=6 \rightarrow 0$. Middle: we calculated the comoving number density of UV-selected galaxies by integrating the UV luminosity functions from Bouwens et al. (2007), Oesch et al. (2010), and Reddy \& Steidel (2009), down to 0.5 $L^{*}$. UV-selected galaxies are bright, star-forming galaxies, such as the LBGs that Adelberger et al. (2005) showed likely host most $\log N_{\mathrm{C} \text { IV }} \geqslant 14$ absorbers at $2 \lesssim z \lesssim 3$. Bottom: assuming that all $W_{\mathrm{r}, 1548} \geqslant 0.6 \AA$ doublets are in UV-selected galaxy halos, we estimated the galaxy-C IV cross-section as a function of redshift with Equation (12). The inferred cross-sections from the SDSS $1.5 \lesssim z \lesssim 4.5$ sample are consistent with the area that Adelberger et al. (2005) and Steidel et al. (2010) measured.

(A color version of this figure is available in the online journal.)

The galaxy-C IV cross-section shows no evolution over the SDSS redshift range within the errors, which are dominated by the $20 \%-60 \%$ uncertainties in $n_{\text {com, UV }}$. Assuming the crosssection is due to a spherical halo that projects with $100 \% \mathrm{C}$ IV covering fraction, the halo radius would be $R_{\text {phys }}=\sqrt{\sigma_{\text {phys }} / \pi} \approx$ $50 \mathrm{kpc}$ for $1.5 \lesssim z \lesssim 4.5$. This distance agrees with Adelberger et al. (2005) and allows for, e.g., a non-unity covering fraction or limiting to brighter UV-selected galaxies, both of which would increase $R_{\text {phys }}$.

There is an approximately 10 -fold increase in $\sigma_{\text {phys }}$ from $z \approx 6 \rightarrow 0$ when we include the $d \mathcal{N}_{\mathrm{C} \text { IV }} / d X$ measurements from Simcoe et al. (2011) and Cooksey et al. (2010), respectively. This increase in the galaxy-C IV cross-section is comparable to that of $d \mathcal{N}_{\mathrm{C} \text { IV }} / d X$ over the same redshift range. Thus, if the $W_{\mathrm{r}} \geqslant 0.6 \AA \mathrm{C}$ IV absorbers were only tracing galaxy halos, the redshift evolution of $d \mathcal{N}_{\mathrm{C} \text { IV }} / d X$ could be solely due to the halos filling up with triply ionized carbon, through some combination of physical growth, increased metallicity, and/or evolution in the ionizing background.

However, the uncertainty in the $z<1$ cross-section estimate is large and consistent with an increase of only two to three compared with the $z \approx 6$ value. In this case, the roughly orderof-magnitude increase in $d \mathcal{N}_{\text {CIV }} / d X$ could equally be due to modest increases in $n_{\text {com, UV }}$ and $\sigma_{\text {phys }}$.

We emphasize that the preceding discussion assumes all $W_{\mathrm{r}} \geqslant$ $0.6 \mathrm{C}$ IV systems at $0<z<6$ are only found in the circumgalactic media of UV-selected galaxies. Any intergalactic C IV contribution to $d \mathcal{N}_{\mathrm{C} \text { IV }} / d X$ at any redshift would cause us to overestimate the galaxy-C IV cross-section.

Evolution in the ultraviolet background (UVB) possibly explains the seeming spike in $\sigma_{\text {phys }}$ at $z \approx 5$. Simcoe $(2011)$ showed that C IV is a preferred, if not dominant, transition of carbon at $z=4.3$ compared to lower redshift, assuming the UVB of Haardt \& Madau (2001) or Faucher-Giguère et al. (2009). Though the redshift window of this effect is small $(\Delta z \approx 0.5)$, the increase in C IV-ionizing photons would increase the $\mathrm{C}$ IV cross-section of UV-selected galaxies and may explain the spike in $d \mathcal{N}_{\mathrm{C} \text { IV }} / d X$ and $\sigma_{\text {phys }}$. In general, triply ionized carbon increasingly becomes a disfavored transition of carbon with decreasing redshift, given a standard model of the UVB (Oppenheimer \& Davé 2008; Simcoe 2011). The UVB likely dominates the ionization flux at the expected tens of kiloparsecs from the host galaxies. Thus, the increasing number of $\mathrm{C}$ IV absorbers toward lower redshift indicates an increasing enrichment of the gas, to more than compensate for the decreasingly favored triply ionized state.

$\mathrm{C}$ IV absorption could also be a galactic wind signature (see Steidel et al. 2010). However, the $d \mathcal{N}_{\text {C IV }} / d X$ redshift evolution does not mimic the evolution of $d \mathcal{N}_{\mathrm{Mg}_{\text {II }}} / d X$ for $W_{\mathrm{r}, 2796} \gtrsim 1 \AA$ absorbers, and these strong $\mathrm{Mg}$ II doublets are often analyzed as wind tracers (Weiner et al. 2009; Rubin et al. 2010; Bordoloi et al. 2011; but also see Gauthier et al. 2010 and Kacprzak et al. 2011). Strong Mg II absorbers evolve strongly with redshift by increasing with increasing redshift (Nestor et al. 2005; Prochter et al. 2006), peaking at $z \approx 3$, and then decreasing at higher redshifts (Matejek \& Simcoe 2012). The latter study showed that the evolution in $d \mathcal{N}_{\mathrm{Mg}_{\text {II }}} / d X$ tracks the cosmic star formation rate (Bouwens et al. 2010), using the scaling relation of Ménard et al. (2011), reinforcing the idea that strong Mg II absorbers arise in galactic winds.

We see no decrease at lower redshifts for strong C IV absorbers (see Figure 9). If strong $\mathrm{CIV}_{\mathrm{IV}}$ and $\mathrm{Mg}$ II absorbers were both tracing winds, then, to account for their different redshift evolution, either they must probe outflows in different ways or strong $\mathrm{C}$ IV absorption traces an additional medium, such as halo gas, that contributes significantly to its $d \mathcal{N}_{\mathrm{C} \text { IV }} / d X$ evolution.

\subsection{IV Mass Density}

The C IV doublet redshifts into optical passbands for $1.5 \lesssim$ $z \lesssim 5.5$ and has been observed extensively with large, groundbased telescopes (Songaila 2001; Boksenberg et al. 2003; Schaye et al. 2003; Scannapieco et al. 2006; D'Odorico et al. 2010). Surveys of ultraviolet quasar spectra taken with $H S T$ cover the $z<1$ C IV systems (Danforth \& Shull 2008; Cooksey et al. 2010). More recently, improved infrared spectrographs have pushed C IV surveys to $z \approx 6$ (Ryan-Weber et al. 2009; Becker et al. 2009; Simcoe et al. 2011). Typically these studies have focused on the evolution of $\Omega_{\mathrm{C}_{\text {IV }}}$, the $\mathrm{C}_{\text {IV }}$ mass density relative to the closure density. The first moment of the column density frequency distribution $f\left(N_{\mathrm{C}_{\text {IV }}}\right)$ is related to $\Omega_{\mathrm{C}_{\text {IV }}}$ as 
follows:

$$
\Omega_{\mathrm{C} \text { IV }}=\frac{H_{0} m_{\mathrm{C}}}{c \rho_{c, 0}} \int_{N_{\min }}^{N_{\max }} f\left(N_{\mathrm{C}_{\text {IV }}}\right) N_{\mathrm{C} \text { IV }} d N_{\mathrm{C} \text { IV }},
$$

where $H_{0}=71.9 \mathrm{~km} \mathrm{~s}^{-1} \mathrm{Mpc}^{-1}$ is the Hubble constant today; $m_{\mathrm{C}}=2 \times 10^{-23} \mathrm{~g}$ is the mass of the carbon atom; $c$ is the speed of light; and the critical density $\rho_{c, 0}=3 H_{0}^{2}(8 \pi G)^{-1}=$ $9.77 \times 10^{-30} \mathrm{~g} \mathrm{~cm}^{-3}$ for our assumed Hubble constant.

The earliest (optical) studies found that $\Omega_{\mathrm{C} \text { IV }}$ was relatively constant for $2 \lesssim z \lesssim 4.5$ (Songaila 2001, 2005; Boksenberg et al. 2003; Schaye et al. 2003; Scannapieco et al. 2006). Cooksey et al. (2010) showed that $\Omega_{\mathrm{C} \text { IV }}$ increased by at least a factor of four from $z \approx 3$ to $z<1$. Using better optical spectra, D'Odorico et al. (2010) found that $\Omega_{\text {CIV }}$ increased smoothly from $z=3$ to $z=1.5$ and mapped well onto the $z<1$ values.

However, all these studies measured a power-law shape for the $f\left(N_{\mathrm{C}_{\text {IV }}}\right)$, which formally corresponds to an infinite C IV mass density. These surveys were limited to small numbers of available sightlines, typically less than 50 . The rarest systems are the strongest, and these dominate the mass density measurement when the distribution is a power law. Hence the small-number statistics on the strongest $C_{\text {IV }}$ absorbers limited the quoted $\Omega_{\text {CIV }}$ to be for $12 \lesssim \log N_{\text {CIV }} \lesssim 15$, with one survey pushing to $\log N_{\text {CIV }} \approx 16$ (Scannapieco et al. 2006). Indeed, scaling the Scannapieco et al. (2006) $\Omega_{\text {CIV }}$ to $\log N_{\text {CIV }} \leqslant 15$ reduces their value by $45 \%$ (Cooksey et al. 2010).

Our analysis, however, provides good statistics on the rare, strong systems that dominate the SDSS sample. The observed $\Omega_{\text {CIV }}$ can be approximated by the sum of the detected C IV absorbers (Lanzetta et al. 1991). The total column density in a given redshift bin is simply

$$
\begin{aligned}
& N_{\text {tot }}=\sum_{\mathcal{N}_{\text {obs }}} N_{\text {C IV }} \\
& \sigma_{N_{\text {tot }}}^{2}=\sigma_{\mathcal{N}_{\text {obs }}}^{2}+\sum_{\mathcal{N}_{\text {obs }}} \sigma_{N_{\text {CIV }}}^{2},
\end{aligned}
$$

where, again, we factor in the counting uncertainty for the number of detections $\sigma_{\mathcal{N}_{\text {obs }}}$. Then, we estimated the mass density relative to the critical density as

$$
\begin{aligned}
\Omega_{\mathrm{C} \text { IV }} & =\frac{H_{0} m_{\mathrm{C}}}{c \rho_{c, 0}} \frac{N_{\mathrm{tot}}}{\left\langle\Delta X\left(W_{\mathrm{r}}\right)\right\rangle} \\
\sigma_{\Omega}^{2} & =\Omega_{\mathrm{CIV}}^{2}\left(\left(\frac{\sigma_{N_{\mathrm{tot}}}}{N_{\mathrm{tot}}}\right)^{2}+\left(\frac{\sigma_{\langle\Delta X\rangle}}{\left\langle\Delta X\left(W_{\mathrm{r}}\right)\right\rangle}\right)^{2}\right) .
\end{aligned}
$$

Since the completeness corrections were compiled in equivalent width space, we used the median $\Delta X\left(W_{\mathrm{r}}\right)$ available to each of the absorbers in the given bin, and the error was the standard deviation of the detections. The small scaling to account for the accepted false-positive distribution follows that of the frequency distribution (Equation (8)).

In Figure 13, we plot $\Omega_{\mathrm{C} \text { IV }}$ over redshift from several studies for $\log N_{\mathrm{C} \text { IV }} \geqslant 14$, adjusted for the new limits and cosmology (see Cooksey et al. 2010 for details). The direct SDSS measurements are lower limits since we have predominately saturated absorbers and use the AODM to estimate column densities. However, we can still exclude values below the limits, including the Songaila (2001) measurements at $z<3 .{ }^{11}$ This reinforces

\footnotetext{
11 Schaye et al. (2003) and Songaila (2005) used pixel optical depth methods to also measure $\Omega_{\mathrm{C} \text { IV }}$, but it is unclear how best to compare their values to our traditional quasar absorption-line study. For this reason, we omit discussion of their results.
}

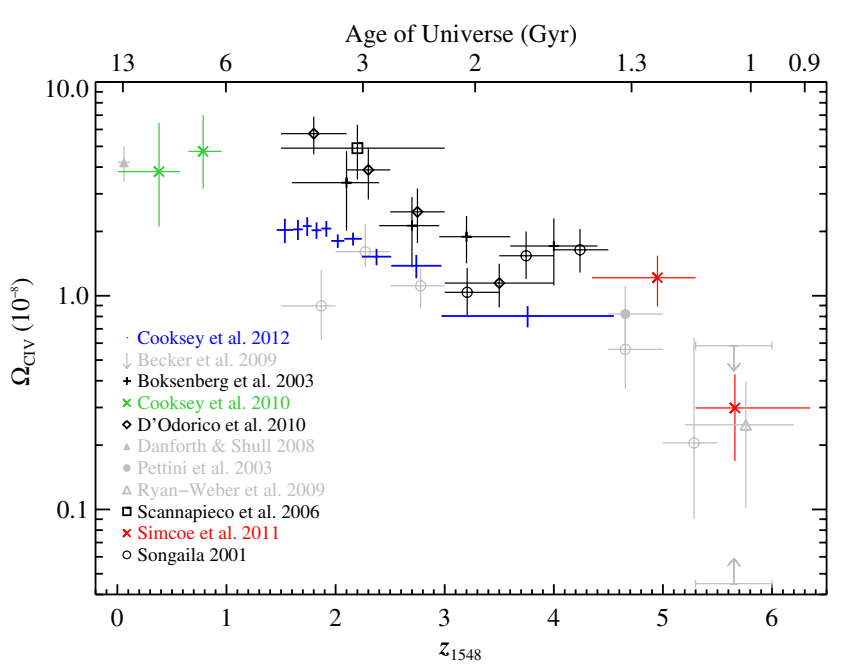

Figure 13. Redshift evolution of $\Omega_{\mathrm{C}_{\text {IV }}}$ for $\log N_{\mathrm{C}_{\text {IV }}} \geqslant 14$ absorbers. The SDSS apparent optical depth values are lower limits (blue), since most systems are saturated. However, these lower limits firmly exclude the $z<3$ measurements from Songaila (2001), which have been grayed out with the other superseded measurements, and support the result that $\Omega_{\mathrm{C} \text { IV }}$ smoothly increases from $z \approx 3$ to $z=1.5$ from D'Odorico et al. (2010).

(A color version of this figure is available in the online journal.)

the D'Odorico et al. (2010) result that $\Omega_{\text {CIV }}$ smoothly increases. In an upcoming paper, we will be combining the D'Odorico et al. (2010) and current data sets to fit $f\left(N_{\text {CIV }}\right)$ and measure $\Omega_{\mathrm{C}_{\text {IV }}}$ for $\log N_{\mathrm{C}_{\text {IV }}} \gtrsim 12$ via Equation (13).

\section{SUMMARY}

We have completed the largest CIV survey to date by leveraging the SDSS DR7 quasar catalog (Schneider et al. 2010). In 26,030 sightlines with $z_{\mathrm{QSO}} \geqslant 1.7$ and $\langle\mathrm{S} / \mathrm{N}\rangle \geqslant 4 \mathrm{pixel}^{-1}$, we identified $14,772 \mathrm{C}$ IV systems at least $5000 \mathrm{~km} \mathrm{~s}^{-1}$ blueward of the quasar. The entire doublet catalog and other tools for analysis (e.g., completeness grids) are made available for the community. ${ }^{12}$

The bulk of the C IV survey was automated, from continuum fitting to candidate selection. We visually verified all doublets in the final catalog. Our Monte Carlo completeness tests included the effects of the automated algorithms, user bias, and accepted false positives. We analyzed the sample as a whole and in 10 redshift bins of roughly 1500 doublets each. We also constructed and analyzed other subsamples as needed, for specific comparisons to published studies.

The equivalent width frequency distribution $f\left(W_{\mathrm{r}}\right)$ is described well by an exponential model. The best-fit parameters evolved smoothly with redshift, with the largest change arising in the normalization. The parameter evolution follows the trend of increasing $d \mathcal{N}_{\mathrm{C} \text { IV }} / d X$ with increasing redshift-a $2.37 \pm 0.09$ increase over $z=4.55 \rightarrow \approx 1.74$. For $1.46 \leqslant z<1.96$, $d \mathcal{N}_{\mathrm{C} \text { IV }} / d X \approx 0.34$.

In the region of overlap, the SDSS $f\left(W_{\mathrm{r}}\right)$ agree well with the published column density frequency distributions $f\left(N_{\text {CIV }}\right)$ from smaller, high-resolution, high-S/N surveys (Songaila 2001; D'Odorico et al. 2010). We converted $f\left(N_{\mathrm{C} \text { IV }}\right)$ to $f\left(W_{\mathrm{r}}\right)$ assuming the linear curve of growth. The published $f\left(N_{\mathrm{C} \text { IV }}\right)$ distributions were best fit by a power-law formalism. Thus, combined with the exponential nature of the strong end of $f\left(W_{\mathrm{r}}\right)$, we at last see an exponential cutoff in the equivalent width distribution that

\footnotetext{
12 See http://igmabsorbers.info/.
} 
has not been previously characterized because of small sample sizes. The location of this cutoff sets the high-end range for C IV absorbers and formally leads to convergence in the cosmic C IV mass density.

In this paper, we joined our large sample of strong absorbers to the fits of weak absorbers obtained at high resolution. In a forthcoming paper, we will perform a joint analysis of our SDSS sample with the high-resolution data of D'Odorico et al. (2010) to fit clean forms to the full range of $f\left(N_{\mathrm{C}_{\text {IV }}}\right)$. This will allow us to improve our understanding of the C IV mass density evolution, where currently our SDSS study only yields lower limits for $\log N_{\mathrm{C}_{\text {IV }}} \geqslant 14$ systems.

The comoving line density $d \mathcal{N}_{\mathrm{C} \text { IV }} / d X$ smoothly increases, approximately, 10-fold from $z=6 \rightarrow 0$ for $W_{\mathrm{r}, 1548} \geqslant 0.6 \AA$ absorbers. Physically, this means that the physical cross-section $\left(\sigma_{\text {phys }}\right)$ and/or comoving number density of C IV absorbing clouds $\left(n_{\text {com }}\right)$ has increased steadily over time, since $d \mathcal{N}_{\text {C IV }} / d X$ is proportional to their product. We estimated the projected C IV-absorbing cross-section of UV-selected galaxies to be $R_{\text {phys }} \approx 50 \mathrm{kpc}$, by assuming all the SDSS $W_{\mathrm{r}} \geqslant 0.6 \AA$ absorbers arise in $\geqslant 0.5 L^{*}$ galaxy halos, which is in good agreement with Adelberger et al. (2005) and Steidel et al. (2010).

This is the first paper in a series on various metal lines in the SDSS DR7 quasar spectra, which will provide a comprehensive and uniform collection of catalogs for future analysis and detailed comparison with cosmological hydrodynamic simulations.

We thank J. Harker, D. Ogden, J. Pritchard, and P. Creasey for their programming help and P. Jonsson for productive discussions regarding statistics and programming. We gratefully acknowledge the vital role of the Adam J. Burgasser Endowed Chair. The current study was funded largely by the National Science Foundation Astronomy \& Astrophysics Postdoctoral Fellowship (AST-1003139) and in part by the MIT Department of Physics and the Alfred P. Sloan Foundation Research Fellowship.

Funding for the SDSS and SDSS-II has been provided by the Alfred P. Sloan Foundation, the Participating Institutions, the National Science Foundation, the U.S. Department of Energy, the National Aeronautics and Space Administration, the Japanese Monbukagakusho, the Max Planck Society, and the Higher Education Funding Council for England. The SDSS Web site is http://www.sdss.org/.

The SDSS is managed by the Astrophysical Research Consortium for the Participating Institutions. The Participating Institutions are the American Museum of Natural History, Astrophysical Institute Potsdam, University of Basel, University of Cambridge, Case Western Reserve University, University of Chicago, Drexel University, Fermilab, the Institute for Advanced Study, the Japan Participation Group, Johns Hopkins University, the Joint Institute for Nuclear Astrophysics, the Kavli Institute for Particle Astrophysics and Cosmology, the Korean Scientist Group, the Chinese Academy of Sciences (LAMOST), Los Alamos National Laboratory, the Max-PlanckInstitute for Astronomy (MPIA), the Max-Planck-Institute for Astrophysics (MPA), New Mexico State University, Ohio State University, University of Pittsburgh, University of Portsmouth, Princeton University, the United States Naval Observatory, and the University of Washington.

Facility: Sloan

\section{REFERENCES}

Abazajian, K. N., Adelman-McCarthy, J. K., Agüeros, M. A., et al. 2009, ApJS, 182,543

Adelberger, K. L., Shapley, A. E., Steidel, C. C., et al. 2005, ApJ, 629, 636

Barlow, T. A., \& Tytler, D. 1998, AJ, 115, 1725

Becker, G. D., Rauch, M., \& Sargent, W. L. W. 2009, ApJ, 698, 1010

Boksenberg, A., Sargent, W. L. W., \& Rauch, M. 2003, arXiv:astro-ph/0307557

Bordoloi, R., Lilly, S. J., Knobel, C., et al. 2011, ApJ, 743, 10

Bouwens, R. J., Illingworth, G. D., Franx, M., \& Ford, H. 2007, ApJ, 670, 928

Bouwens, R. J., Illingworth, G. D., González, V., et al. 2010, ApJ, 725, 1587

Chen, H.-W., Lanzetta, K. M., \& Webb, J. K. 2001, ApJ, 556, 158

Cherinka, B., \& Schulte-Ladbeck, R. E. 2011, AJ, 142, 122

Connolly, A. J., \& Szalay, A. S. 1999, AJ, 117, 2052

Cooksey, K. L., Thom, C., Prochaska, J. X., \& Chen, H. 2010, ApJ, 708,868

Danforth, C. W., \& Shull, J. M. 2008, ApJ, 679, 194

D’Odorico, V., Calura, F., Cristiani, S., \& Viel, M. 2010, MNRAS, 401, 2715

Ellison, S. L., Songaila, A., Schaye, J., \& Pettini, M. 2000, AJ, 120,1175

Faucher-Giguère, C., Lidz, A., Zaldarriaga, M., \& Hernquist, L. 2009, ApJ, 703,1416

Fox, A. J., Savage, B. D., \& Wakker, B. P. 2005, AJ, 130, 2418

Frank, S., Mathur, S., Pieri, M. M., \& York, D. G. 2010, AJ, 140, 835

Gauthier, J.-R., Chen, H.-W., \& Tinker, J. L. 2010, ApJ, 716, 1263

Haardt, F., \& Madau, P. 2001, in Clusters of Galaxies and the High Redshift Universe Observed in X-rays, ed. D. M. Neumann \& J. T. V. Tran (Saclay: CEA), 64

Hewett, P. C., \& Wild, V. 2010, MNRAS, 405, 2302

Kacprzak, G. G., Churchill, C. W., Barton, E. J., \& Cooke, J. 2011, ApJ, 733,105

Komatsu, E., Dunkley, J., Nolta, M. R., et al. 2009, ApJS, 180, 330

Lanzetta, K. M., McMahon, R. G., Wolfe, A. M., et al. 1991, ApJS, 77, 1

Martin, C. L., Scannapieco, E., Ellison, S. L., et al. 2010, ApJ, 721, 174

Matejek, M. S., \& Simcoe, R. A. 2012, ApJ, 761, 112

Ménard, B., Wild, V., Nestor, D., et al. 2011, MNRAS, 417, 801

Nestor, D. B., Turnshek, D. A., \& Rao, S. M. 2005, ApJ, 628, 637

Oesch, P. A., Bouwens, R. J., Carollo, C. M., et al. 2010, ApJL, 725, 150

Oppenheimer, B. D., \& Davé, R. 2008, MNRAS, 387, 577

Pieri, M. M., Frank, S., Mathur, S., et al. 2010a, ApJ, 716, 1084

Pieri, M. M., Frank, S., Weinberg, D. H., Mathur, S., \& York, D. G. 2010b, ApJL, 724, 69

Prochaska, J. X., Herbert-Fort, S., \& Wolfe, A. M. 2005, ApJ, 635, 123

Prochaska, J. X., O’Meara, J. M., \& Worseck, G. 2010, ApJ, 718, 392

Prochaska, J. X., Weiner, B., Chen, H.-W., Cooksey, K. L., \& Mulchaey, J. S. 2011, ApJS, 193, 28

Prochter, G. E., Prochaska, J. X., \& Burles, S. M. 2006, ApJ, 639, 766

Reddy, N. A., \& Steidel, C. C. 2009, ApJ, 692, 778

Richards, G. T., Fan, X., Newberg, H. J., et al. 2002, AJ, 123, 2945

Rubin, K. H. R., Weiner, B. J., Koo, D. C., et al. 2010, ApJ, 719, 1503

Ryan-Weber, E. V., Pettini, M., \& Madau, P. 2006, MNRAS, 371, L78

Ryan-Weber, E. V., Pettini, M., Madau, P., \& Zych, B. J. 2009, MNRAS, 395,1476

Savage, B. D., \& Sembach, K. R. 1991, ApJ, 379, 245

Scannapieco, E., Pichon, C., Aracil, B., et al. 2006, MNRAS, 365, 615

Schaye, J., Aguirre, A., Kim, T.-S., et al. 2003, ApJ, 596, 768

Schaye, J., Carswell, R. F., \& Kim, T.-S. 2007, MNRAS, 379, 1169

Schneider, D. P., Richards, G. T., Hall, P. B., et al. 2010, AJ, 139, 2360

Shen, Y., Richards, G. T., Strauss, M. A., et al. 2011, ApJS, 194, 45

Simcoe, R. A. 2006, ApJ, 653, 977

Simcoe, R. A. 2011, ApJ, 738, 159

Simcoe, R. A., Burgasser, A. J., Bochanski, J. J., et al. 2010, Proc. SPIE, 7735, 773514

Simcoe, R. A., Cooksey, K. L., Matejek, M., et al. 2011, ApJ, 743, 21

Songaila, A. 2001, ApJL, 561, 153

Songaila, A. 2005, AJ, 130, 1996

Steidel, C. C. 1990, ApJS, 72, 1

Steidel, C. C., Erb, D. K., Shapley, A. E., et al. 2010, ApJ, 717, 289

Weiner, B. J., Coil, A. L., Prochaska, J. X., et al. 2009, ApJ, 692, 187

Wild, V., Hewett, P. C., \& Pettini, M. 2006, MNRAS, 367, 211

Wilson, E. B. 1927, JASA, 22, 209

Worseck, G., \& Prochaska, J. X. 2011, ApJ, 728, 23

Yip, C. W., Connolly, A. J., Vanden Berk, D. E., et al. 2004, AJ, 128, 2603

York, D. G., Adelman, J., Anderson, J. E., Jr., et al. 2000, AJ, 120, 1579 